\title{
Existence and uniqueness of bounded weak solutions for some nonlinear parabolic problems
}

\section{Weilin Zou* and Juan Li}

\section{"Correspondence:} zwl267@aliyun.com; zwl267@163.com

College of Mathematics and Information Science, Nanchang Hangkong University, Nanchang, 330063, China

\begin{abstract}
In this paper we study a class of nonlinear parabolic problems with $p(x, t)$ growth conditions. We prove the existence and uniqueness of bounded solutions to such a problem, with less constraint to $p(x, t)$. Our results are generalizations of the corresponding results in the constant exponent case.
\end{abstract}

MSC: 34A12; 35K55; 35A01; 35A02

Keywords: nonlinear parabolic equation; variable exponents; $p(x, t)$ growth; $L^{\infty}$ estimates

\section{Introduction}

Our main goal is to prove the existence and uniqueness of solutions to the following nonlinear parabolic problem:

$$
(P) \begin{cases}\frac{\partial u}{\partial t}-\operatorname{div} a(x, t, u, \nabla u)-\operatorname{div} \phi(u)+g(x, t, u)=f-\operatorname{div} F & \text { in } Q, \\ u=0 & \text { on } \partial \Omega \times(0, T), \\ u(x, 0)=u_{0} & \text { in } \Omega,\end{cases}
$$

where $\Omega$ is an open bounded subset of $\mathbb{R}^{N}(N \geq 1)$ with Lipschitz boundary $\partial \Omega, T$ is a positive constant, $u_{0} \in L^{\infty}(\Omega), Q=\Omega \times(0, T)$ with the lateral boundary $\partial \Omega \times(0, T)$.

Here, we make the following assumptions on $a, \phi, g, f$, and $F$ :

$\left(\mathrm{H}_{1}\right)$ The function $a: Q \times \mathbb{R} \times \mathbb{R}^{N}$ is a Carathéodory function and there exist a continuous function $p: \bar{Q} \rightarrow(1,+\infty)$ and a positive constant $\alpha$ such that

$$
a(x, t, s, \xi) \xi \geq \alpha|\xi|^{p(x, t)}, \quad \text { a.e. }(x, t) \in Q, \forall s \in \mathbb{R} \text { and } \forall \xi \in \mathbb{R}^{N} \text {. }
$$

$\left(\mathrm{H}_{2}\right)$ There exist a continuous function $b$ from $\mathbb{R}^{+}$into $\mathbb{R}^{+}$and a nonnegative function $c \in L^{p^{\prime}(x, t)}(Q)$ such that

$$
|a(x, t, s, \xi)| \leq b(|s|)\left[|\xi|^{p(x, t)-1}+c(x, t)\right], \quad \text { a.e. }(x, t) \in Q, \forall s \in \mathbb{R} \text { and } \forall \xi \in \mathbb{R}^{N}
$$

$\left(\mathrm{H}_{3}\right)(a(x, t, s, \xi)-a(x, t, s, \zeta)) \cdot(\xi-\zeta)>0$ holds for almost every $(x, t) \in Q$ and for every $\xi, \zeta \in \mathbb{R}^{N}$ with $\xi \neq \zeta$.

(c) 2015 Zou and Li; licensee Springer. This article is distributed under the terms of the Creative Commons Attribution 4.0 International License (http://creativecommons.org/licenses/by/4.0/), which permits unrestricted use, distribution, and reproduction in any medium, provided you give appropriate credit to the original author(s) and the source, provide a link to the Creative Commons license, and indicate if changes were made. 
$\left(\mathrm{H}_{4}\right) g: Q \times \mathbb{R}$ is a Carathéodory function, satisfying $\sup _{|s| \leq n}|g(\cdot, s)|=h_{n}(\cdot) \in L^{1}(Q)$ and

$$
g(x, t, s) s \geq 0, \quad \text { for a.e. }(x, t) \in Q \text { and } \forall s \in \mathbb{R} \text {. }
$$

$\left(\mathrm{H}_{5}\right)$ The function $\phi$ is continuous on $\mathbb{R}$ with values in $\mathbb{R}^{N}$.

$\left(\mathrm{H}_{6}\right) f \in L^{q(x, t)}(Q)$ and $F \in\left(L^{q(x, t)\left(p^{-}\right)^{\prime}}(Q)\right)^{N}$, where $q^{-}>\max \left\{1+\frac{N}{p^{-}}, 2\right\}$.

As we have seen, problem $(P)$ includes parabolic equation which is nonlinear with respect to the gradient of the solution, and with variable exponents of nonlinearity. Thus it is natural to solve problem $(P)$ under the framework of Sobolev spaces with variable exponents. The problem we study here is closely related to the model of electro-rheological fluids (see [1-3]). For more applications, we refer the reader to [4-17].

In the case of $p$ and $q$ are two constants, the existence and regularity of the solutions to problem $(P)$ have been intensively studied by many authors. We refer the reader to the bibliography [18] and references therein. Especially, it is well known that problem $(P)$ have a weak solution belongs to $L^{\infty}(Q)$, provided that $q>\max \left\{1+\frac{N}{p}, 2\right\}$.

In the stationary case and $p=p(x)$, there have been several results concerning the existence, uniqueness and regularity of entropy or renormalized solutions to such problems with $q=1$ and $F \equiv 0$; see [7] and [8] for example. More precisely, in [7], it is assumed that $p(x)$ belongs to $W^{1, \infty}(\Omega)$; in [8], it is assumed that $p(x) \in C(\bar{\Omega})$ satisfies the log-continuity condition. We also remark that the existence of bounded weak solutions to this type of problems have been studied in [9-11], assuming that $p(x) \in C(\bar{\Omega})$.

Recently some papers appeared in the case of parabolic problems with non-standard growth. When $p=p(x) \in C(\bar{\Omega})$ satisfies the log-continuity condition, the existence and uniqueness of an entropy solution to problem $(P)$ without lower order terms were proved in [12], under the assumption $f \in L^{1}(Q)$ and $F \equiv 0$. When $p=p(x)$ only belongs to $C(\bar{\Omega})$ with $p^{-}>1, \mathrm{M}$. Bendahmane et al. have also proved the existence and uniqueness of renormalized solutions, by the semigroup approach; see [13]. If $p \in C(\bar{\Omega})$ and $p^{-}>2$, the existence of weak solutions to problem $(P)$ is proved in [14], for $\phi=0$ and $F \equiv 0$.

When $p$ is Lipschitz continuous with respect to the space variables and $\frac{\beta}{2}$-Hölder continuous with respect to time, Acerbi et al. [15] studied the regularity results for parabolic systems without lower order terms and $f, F \equiv 0$. As $p=p(x, t) \in C(\bar{Q})$ satisfies the logcontinuity condition and $F \equiv 0$, Antontsev and Shmarev [16] studied the existence of solutions of similar problems with anisotropic parabolic equation. Moreover, it is worth to mention that Alkhutov and Zhikov [17] obtained the existence results without any assumption on the regularity of the exponent, if the terms $g, \phi, F \equiv 0$.

The main idea of this paper relies on $[13,14,16,18,19]$. Using Galerkin's approximation technique, we shall prove the existence and uniqueness of bounded solutions to problem $(P)$ (Theorem 3.1 and Theorem 4.1), which generalizes the corresponding results in the constant exponents. In order to prove Theorem 3.1, a key result (Lemma 3.2) about an $L^{\infty}$ estimate for solution to problem $(P)$ is proved.

This paper is organized as follows: in Section 2 we recall some basic notations and properties of Sobolev spaces with variable exponents; in Section 3, we prove the existence of solutions to problem $(P)$; in Section 4, we give the proof of uniqueness of solutions to problem $(P)$. 


\section{Some preliminaries and notations}

In what follows, we recall some definitions and basic properties of the generalized Lebesgue space $L^{p(x)}(\Omega)$ and the generalized Sobolev spaces $W^{1, p(x)}(\Omega)$ (see [20] and [21], etc.).

Set $C_{+}(\bar{\Omega})=\left\{h \in C(\bar{\Omega}): \inf _{x \in \bar{\Omega}} h(x)>1\right\}$. For any $h \in C_{+}(\bar{\Omega})$, we define

$$
h^{+}=\sup _{x \in \bar{\Omega}} h(x) \quad \text { and } \quad h^{-}=\inf _{x \in \bar{\Omega}} h(x)
$$

For any $p \in C_{+}(\bar{\Omega})$, we define the variable exponent Lebesgue spaces $L^{p(x)}(\Omega)$ to consist of all measurable functions such that the modular

$$
\rho_{p}(f):=\int_{\Omega}|f(x)|^{p(x)} \mathrm{d} x
$$

is finite, endowed with the Luxemburg norm

$$
\|u\|_{L^{p(x)}(\Omega)}=\inf \left\{\lambda>0: \int_{\Omega}\left|\frac{u(x)}{\lambda}\right|^{p(x)} \mathrm{d} x \leq 1\right\} .
$$

Lemma 2.1 (1) The space $L^{p(x)}(\Omega)$ is a separable and reflexive Banach space, and its dual space is isomorphic to $L^{p^{\prime}(\cdot)}(\Omega)$, where $\frac{1}{p(x)}+\frac{1}{p^{\prime}(x)}=1$. For any $u \in L^{p(x)}(\Omega)$ and $v \in L^{p^{\prime}(\cdot)}(\Omega)$,

$$
\left|\int_{\Omega} u v \mathrm{~d} x\right| \leq\left(\frac{1}{p^{-}}+\frac{1}{\left(p^{\prime}\right)^{-}}\right)\|u\|_{L^{p(x)}(\Omega)}\|v\|_{L^{p^{\prime}(\cdot)(\Omega)}} .
$$

(2) If $p_{1}, p_{2} \in C_{+}(\bar{\Omega})$ with $p_{1}(x) \leq p_{2}(x)$, for any $x \in \Omega$, then there exists the continuous embedding $L^{p_{2}(\cdot)}(\Omega) \hookrightarrow L^{p_{1}(\cdot)}(\Omega)$, whose norm does not exceed $|\Omega|+1$.

(3) $C_{0}^{\infty}(\Omega)$ is dense in $L^{p(x)}(\Omega)$.

(4) For any $u \in L^{p(x)}(\Omega)$, we have

$$
\min \left\{\|u\|_{L^{p(x)}(\Omega)}^{p^{-}}\|u\|_{L^{p(x)}(\Omega)}^{p^{+}}\right\} \leq \int_{\Omega}|u(x)|^{p(x)} \mathrm{d} x \leq \max \left\{\|u\|_{L^{p(x)}(\Omega)}^{p^{-}}\|u\|_{L^{p(x)}(\Omega)}^{p^{+}}\right\} .
$$

(5) Let $\left\{v_{n}\right\} \subseteq L^{p(x)}(\Omega)$ and $v \in L^{p(x)}(\Omega)$, the following statements are equivalent:

(i) $\lim _{n \rightarrow \infty}\left\|v_{n}-v\right\|_{L^{p(x)}(\Omega)}=0$;

(ii) $\lim _{n \rightarrow \infty} \rho_{p}\left(v_{n}-v\right)=0$;

(iii) $v_{n}$ converges to $v$ in measure and $\lim _{n \rightarrow \infty} \rho_{p}\left(v_{n}\right)=\rho_{p}(v)$.

Remark 2.1 Obviously, if $p$ is a constant function, then the variable exponent Lebesgue space coincides with the usual Lebesgue space.

Set

$$
W^{1, p(x)}(\Omega)=\left\{u \in L^{p(x)}(\Omega):|\nabla u| \in L^{p(x)}(\Omega)\right\}
$$

where the norm is defined by

$$
\|u\|_{W^{1, p(x)}(\Omega)}=\|u\|_{L^{p(x)}(\Omega)}+\|\nabla u\|_{L^{p(x)}(\Omega)} .
$$


The space $W^{1, p(x)}(\Omega)$ is called a generalized Sobolev space. By $W_{0}^{1, p(x)}(\Omega)$ we denote the subspace of $W^{1, p(x)}(\Omega)$ which is the closure of $C_{0}^{\infty}(\Omega)$ with respect to the norm (2.2). We denote the dual space of $W_{0}^{1, p(x)}(\Omega)$ by $\left(W_{0}^{1, p(x)}(\Omega)\right)^{\star}$.

Lemma 2.2 (see [21] or [22]) The space $W^{1, p(x)}(\Omega)$ and $W_{0}^{1, p(x)}(\Omega)$ are reflexive Banach spaces. For any $u \in W_{0}^{1, p(x)}(\Omega)$, the Poincaré inequality

$$
\|u\|_{L^{p(x)}(\Omega)} \leq c\|\nabla u\|_{L^{p(x)}(\Omega)}
$$

holds true, where $c$ is a constant depending on $\Omega, N$, and $p$.

Lemma 2.3 (see [20]) Let $p, d \in C_{+}(\bar{\Omega})$ with $p^{+}<N$ and $d(x)<p^{*}(x):=\frac{N p(x)}{N-p(x)}$ almost everywhere in $\Omega$, then there is a continuous and compact imbedding $W_{0}^{1, p(x)}(\Omega) \hookrightarrow \hookrightarrow L^{d(\cdot)}(\Omega)$, and

$$
\|u\|_{L^{d(\cdot)(\Omega)}} \leq \tilde{C}\|\nabla u\|_{L^{p(x)}(\Omega)},
$$

where $\tilde{C}$ depends only on $\Omega, N, p^{+}$, and $d^{+}$.

Remark 2.2 In general, the smooth functions are not dense in $W^{1, p(x)}(\Omega)$ (see [21]). However, if the exponent $p(x)$ is assumed to be log-Hölder continuous, i.e. there exists a positive constant $C$ such that

$$
|p(x)-p(y)| \leq \frac{C}{-\log |x-y|}, \quad \text { for any } x, y \in \Omega \text { with }|x-y| \leq \frac{1}{2},
$$

then the smooth functions are dense in $W^{1, p(x)}(\Omega)$ and $W_{0}^{1, p(x)}(\Omega)=W^{1, p(x)}(\Omega) \cap W_{0}^{1,1}(\Omega)$ (see [20, 21]). Moreover, if $p \in C_{+}(\bar{\Omega})$ satisfies $(2.5)$ and $p^{+}<N$ then the Sobolev embedding holds also for $d(x)=p^{*}(x)$, i.e. $W^{1, p(x)}(\Omega) \hookrightarrow L^{p^{*}(x)}(\Omega)$. As in [13, 23], we do not need these condition to prove our result and will most exclusively work with $p \in C_{+}(\bar{\Omega})$. We also observe that $W_{0}^{1, p(x)}(\Omega)$ is stable by composition with Lipschitz functions, even if for a function $v \in W^{1, p(x)}(\Omega)$ having trace zero does not guarantee that $v \in W_{0}^{1, p(x)}(\Omega)$. In other words, if $L: \mathbb{R} \rightarrow \mathbb{R}$ is Lipschitz continuous such that $L(0)=0$ and $v \in W_{0}^{1, p(x)}(\Omega)$, then $L(v) \in W_{0}^{1, p(x)}(\Omega)$. For more details, one can refer to $[13,23]$ for example.

Now, for any $p \in C_{+}(\bar{Q})$, we define

$$
p^{+}=\sup _{(x, t) \in \bar{Q}} p(x, t) \text { and } \quad p^{-}=\inf _{(x, t) \in \bar{Q}} p(x, t) .
$$

We may also consider the generalized Lebesgue space

$$
L^{p(x, t)}(Q)=\left\{u: Q \rightarrow \mathbb{R} ; u \text { is measurable with } \int_{0}^{T} \int_{\Omega}|u(x, t)|^{p(x, t)} \mathrm{d} x \mathrm{~d} t<\infty\right\},
$$

endowed with the norm

$$
\|u\|_{L^{p(x, t)}(Q)}=\inf \left\{\lambda>0: \int_{0}^{T} \int_{\Omega}\left|\frac{u(x, t)}{\lambda}\right|^{p(x, t)} \mathrm{d} x \mathrm{~d} t \leq 1\right\},
$$

which obviously shares the same type of properties as $L^{p(x)}(\Omega)$. 
We will also use the standard notations for Bochner spaces, i.e., if $q \geq 1$ and $X$ is a Banach space, then $L^{q}(0, T ; X)$ denotes the space of strongly measurable functions $u:(0, T) \rightarrow X$ for which $t \mapsto\|u(t)\|_{X} \in L^{q}(0, T)$. Moreover, $C([0, T] ; X)$ denotes the space of continuous functions $u:[0, T] \rightarrow X$ endowed with the norm $\|u\|_{C([0, T] ;)}:=\max _{t \in[0, T]}\|u(t)\|_{X}$.

For any given $k>0$, the truncation function $T_{k}$ is defined as follows:

$$
T_{k}(s)= \begin{cases}k, & s>k, \\ s, & |s| \leq k, \\ -k, & s<-k .\end{cases}
$$

We use $C\left(\theta_{1}, \theta_{2}, \ldots, \theta_{m}\right)$ to denote positive constants depending only on specified quantities $\theta_{1}, \theta_{2}, \ldots, \theta_{m}$. Throughout this paper, the notation $X^{\star}$ denotes the dual space of a Banach space $X$.

\section{Existence of weak solution to problem $(P)$}

First of all, we shall give the definition of weak solution to problem $(P)$. To do this, we need to introduce the following Banach space:

$$
W(Q)=\left\{u \text { is measurable }: u \in L^{p^{-}}\left(0, T ; W_{0}^{1, p(x, t)}(\Omega)\right) \text { and }|\nabla u| \in L^{p(x, t)}(Q)\right\}
$$

endowed with the norm

$$
\|u\|_{W(Q)}:=\|u\|_{L^{p^{-}}\left(0, T ; W_{0}^{1, p(x, t)}(\Omega)\right)}+\|\nabla u\|_{L^{p(x, t)}(Q)} .
$$

Remark 3.1 The space $W(Q)$ is reflexive and separable. Moreover, there exists an equivalent norm of $W(Q)$ :

$$
\|u\|_{W(Q)}:=\|\nabla u\|_{L^{p(x, t)}(Q)} .
$$

As in [13], we have the following result.

Lemma 3.1 (i) We have the following continuous dense embeddings:

$$
\begin{aligned}
L^{p^{+}}\left(0, T ; W_{0}^{1, p^{+}}(\Omega)\right) & \stackrel{d}{\hookrightarrow} L^{p^{+}}\left(0, T ;\left(W_{0}^{1, p(x, t)}(\Omega)\right)\right) \stackrel{d}{\hookrightarrow} W(Q) \\
& \stackrel{d}{\hookrightarrow} L^{p^{-}}\left(0, T ; W_{0}^{1, p(x, t)}(\Omega)\right) \stackrel{d}{\hookrightarrow} L^{p^{-}}\left(0, T ; W_{0}^{1, p^{-}}(\Omega)\right) .
\end{aligned}
$$

In particular, $\mathscr{D}(Q)$ is dense in $W(Q)$ and

$$
\begin{aligned}
& L^{\left(p^{-}\right)^{\prime}}\left(0, T ;\left(W_{0}^{1, p^{-}}(\Omega)\right)^{\star}\right) \\
& \quad \hookrightarrow L^{\left(p^{-}\right)^{\prime}}\left(0, T ;\left(W_{0}^{1, p(x, t)}(\Omega)\right)^{\star}\right) \hookrightarrow W^{\star}(Q) \\
& \quad \hookrightarrow L^{\left(p^{+}\right)^{\prime}}\left(0, T ;\left(W_{0}^{1, p(x, t)}(\Omega)\right)^{\star}\right) \hookrightarrow L^{\left(p^{+}\right)^{\prime}}\left(0, T ;\left(W_{0}^{1, p^{+}}(\Omega)\right)^{\star}\right) .
\end{aligned}
$$

(ii) If $T \in W^{\star}(Q)$, there exists $f_{0} \in L^{p(x, t)}(Q), F=\left(f_{1}, \ldots, f_{N}\right) \in\left(L^{p(x, t)}(Q)\right)^{N}$ such that $T=$ $f_{0}-\operatorname{div} F$, and

$$
\langle T, v\rangle_{W^{\star}(Q), W(Q)}=\int_{0}^{T} \int_{\Omega} f_{0} v+F \cdot \nabla v \mathrm{~d} x \mathrm{~d} t, \quad \forall v \in W(Q) .
$$


Furthermore, we have

$$
\|T\|_{W^{\star}(Q)}=\max \left\{\left\|f_{i}\right\|_{L^{p(x, t)}(Q)}, i=1, \ldots, n\right\} .
$$

(iii) If $v \in W(Q)$ with $v_{t} \in L^{1}(Q)+W^{\star}(Q)$, then we have $v \in C\left([0, T] ; L^{1}(\Omega)\right)$. Furthermore, if $v \in W(Q) \cap L^{\infty}(Q)$ with $v_{t} \in L^{1}(Q)+W^{\star}(Q)$, then $v \in C\left([0, T] ; L^{2}(\Omega)\right)$.

(iv) If $v \in W(Q) \cap L^{2}\left(0, T ; L^{2}(\Omega)\right)$ with $v_{t} \in W^{\star}(Q)$, then $v \in C\left([0, T] ; L^{2}(\Omega)\right)$.

Proof of Lemma 3.1 The proofs of results (i) and (ii) are similar to [24], the proofs of (iii) and (iv) are similar to [25]. We omit the details here.

Now we give the definition of weak solutions to problem $(P)$.

Definition 3.1 A function $u(x, t) \in W(Q)$ is called a weak solution of problem $(P)$, if $a(x, t, u, \nabla u) \in\left(L^{p^{\prime}(x, t)}(Q)\right)^{N}, \phi(u) \in\left(L^{p^{\prime}(x, t)}(Q)\right)^{N}$, and $g(x, t, u) \in L^{1}(Q)$ such that such that

$$
\frac{\partial u}{\partial t}-\operatorname{div} a(x, t, u, \nabla u)-\operatorname{div} \phi(u)+g(x, t, u)=f-\operatorname{div} F \quad \text { in } \mathscr{D}^{\prime}(Q)
$$

with $\left.u\right|_{t=0}=u_{0}$.

Remark 3.2 Note that if $u$ is a weak solution of problem (P), then $u \in W(Q)$ and $u_{t} \in$ $L^{1}(Q)+W^{\star}(Q)$, so $u \in C\left([0, T] ; L^{1}(\Omega)\right)$. Therefore the initial condition $\left.u\right|_{t=0}=u_{0}$ makes sense.

Remark 3.3 If $u$ is a solution of problem (P), by Remark 3.2, the equality (3.4) reads

$$
\begin{gathered}
\int_{0}^{T}\left\langle\frac{\partial u}{\partial t}, \eta\right\rangle \mathrm{d} t+\int_{0}^{T} \int_{\Omega}[a(x, t, u, \nabla u)+\phi(u)] \nabla \eta \mathrm{d} x \mathrm{~d} t+\int_{0}^{T} \int_{\Omega} g(x, t, u) \eta \mathrm{d} x \mathrm{~d} t \\
=\int_{0}^{T} \int_{\Omega} f \eta \mathrm{d} x \mathrm{~d} t+\int_{0}^{T} \int_{\Omega} F \nabla \eta \mathrm{d} x \mathrm{~d} t, \quad \forall \eta \in W(Q) \cap L^{\infty}(Q),
\end{gathered}
$$

where $\langle\cdot, \cdot \cdot\rangle$ denotes the duality pairing between $W^{\star}(Q)+L^{1}(Q)$ and $W(Q) \cap L^{\infty}(Q)$.

In order to find some estimates for weak solutions and also to get the uniqueness result, the following integration-by-parts-formula is needed (of which the proof will be given in the Appendix):

Lemma 3.2 Let $\varphi: \mathbb{R} \mapsto \mathbb{R}$ be a continuous piecewise $C^{1}$ function such that $\varphi(0)=0$ and $\varphi$ is zero outside a compact set of $\mathbb{R}$. Let us denote $\tilde{\varphi}(s)=\int_{0}^{s} \varphi(r) \mathrm{d} r$. If $u \in W(Q)$ with $\frac{\partial u}{\partial t} \in$ $W^{\star}(Q)+L^{1}(Q)$ and if $\psi \in C^{\infty}(\bar{Q})$, then we have, for any $\tau \in(0, T]$,

$$
\begin{aligned}
\int_{0}^{T}\left\langle\frac{\partial u}{\partial t}, \varphi(u) \chi_{(0, \tau)(t)} \psi\right\rangle \mathrm{d} t= & \left.\int_{\Omega}(\tilde{\varphi}(u) \psi)\right|_{t=\tau} \mathrm{d} x-\left.\int_{\Omega}(\tilde{\varphi}(u) \psi)\right|_{t=0} \mathrm{~d} x \\
& -\int_{0}^{\tau} \int_{\Omega} \frac{\partial \psi}{\partial t} \tilde{\varphi}(u) \mathrm{d} x \mathrm{~d} \tau .
\end{aligned}
$$

From the proof of Lemma 3.2, it is easy to obtain the following conclusion. 
Corollary 3.1 Let $u \in W(Q)$ with $\frac{\partial u}{\partial t} \in W^{\star}(Q)+L^{1}(Q)$. If $\varphi: \mathbb{R} \mapsto \mathbb{R}$ is a continuous function such that $\varphi(u) \in W(Q)$ and $\tilde{\varphi}(u) \in C\left([0, T] ; L^{1}(\Omega)\right)$, then (3.6) holds true.

Theorem 3.1 Let $p \in C_{+}(\bar{Q})$, assume that $\left(\mathrm{H}_{1}\right)-\left(\mathrm{H}_{6}\right)$ hold, then problem $(P)$ admits at least a weak solution $u \in L^{\infty}(Q) \cap C\left(0, T ; L^{2}(\Omega)\right)$.

Before giving the proof Theorem 3.1, we need an $L^{\infty}$ estimate which is stated as follows.

Lemma 3.3 Let $u \in L^{\infty}(Q) \cap C\left([0, T] ; L^{2}(\Omega)\right)$ be a weak solution to problem $(P)$ and suppose that the assumptions of Theorem 3.1 hold true, then there exists a positive constant $M$ such that

$$
\|u\|_{L^{\infty}(Q)} \leq M
$$

where $M$ is a positive constant only depending on $p^{-}, p^{+}, \alpha, N, \Omega,\|f\|_{L^{q^{-}}(\mathrm{Q})},\left\|u_{0}\right\|_{L^{\infty}(\Omega)}$ and $\left\||F|^{\frac{p^{-}}{p^{-}-1}}\right\|_{L^{q^{-}}(Q)}$.

Remark 3.4 It is well known that if $p>1$ is a constant function, then one may obtain an $L^{\infty}$ estimates for $u$ provided that $q>\max \left\{1+\frac{N}{p}, 2\right\}$. The above result is a generalization of the corresponding result in the constant exponent case.

To prove Lemma 3.3, we need the following result, which can be viewed as a generalization of Lemma 4.1 in [18].

Lemma 3.4 Let $Y_{n}, n=0,1,2, \ldots$, be a sequence of positive numbers, satisfying the inequalities

$$
Y_{n+1} \leq c b^{n}\left[Y_{n}^{\beta_{1}}+Y_{n}^{\beta_{2}}+\cdots+Y_{n}^{\beta_{j}}\right]
$$

where $j$ is a positive integer, $c, b>1$ and $\beta_{i}$ are given positive numbers with

$$
\beta=\min _{1 \leq i \leq j}\left\{\beta_{i}\right\}>1
$$

Assuming that

$$
Y_{0} \leq(c j)^{\frac{-1}{\beta-1}} b^{\frac{-1}{(\beta-1)^{2}}}
$$

then $\lim _{n \rightarrow \infty} Y_{n}=0$.

Proof of Lemma 3.4 The proof is by induction as in [18]. However, the details of the proof are omitted. In order to be complete and self-contained, let us briefly explain the argument.

In view of (3.9), we get $0<Y_{0}<1$. Thus, by (3.8) and (3.9), we get

$$
Y_{1} \leq(c j)^{\frac{-1}{\mu}} b^{-\frac{1+\mu}{\mu^{2}}}
$$

where $\mu=\beta-1$. 
Obviously $0<Y_{1}<1$, thus, using (3.8) again, we have

$$
Y_{2} \leq(c j)^{\frac{-1}{\mu}} b^{-\frac{1+2 \mu}{\mu^{2}}}
$$

By induction, we easily find that

$$
Y_{n} \leq(c j)^{\frac{-1}{\mu}} b^{-\frac{1+n \mu}{\mu^{2}}} .
$$

Letting $n$ tend to infinity in the above inequality, we obtain the desired result immediately.

Proof of Lemma 3.3 Set $\bar{M}=\|u\|_{L^{\infty}(Q)}$. Without loss of generality, we may assume $\bar{M}>$ $2\left\|u_{0}\right\|_{L^{\infty}(\Omega)}$. For every $k$ with $\max \left\{\left\|u_{0}\right\|_{L^{\infty}(\Omega)}, \bar{M}-1\right\} \leq k \leq \bar{M}$ and any given $\tau \in(0, T)$, taking $\eta(x, t)=\operatorname{sign} u(|u|-k)_{+} \chi_{(0, \tau)}(t)$ as a test function in problem $(P)$, we obtain

$$
\begin{aligned}
\left\langle\frac{\partial u}{\partial t}\right. & \left., \operatorname{sign} u(|u|-k)_{+} \chi_{(0, \tau)}(t)\right\rangle+\int_{0}^{\tau} \int_{\Omega} a(x, t, u, \nabla u) \nabla\left(\operatorname{sign} u(|u|-k)_{+}\right) \mathrm{d} x \mathrm{~d} t \\
& +\int_{0}^{\tau} \int_{\Omega} \phi(u) \nabla\left(\operatorname{sign} u(|u|-k)_{+}\right) \mathrm{d} x \mathrm{~d} t+\int_{0}^{\tau} \int_{\Omega} g(x, t, u) \operatorname{sign} u(|u|-k)_{+} \mathrm{d} x \mathrm{~d} t \\
= & \int_{0}^{\tau} \int_{\Omega} f \operatorname{sign} u(|u|-k)_{+} \mathrm{d} x \mathrm{~d} t+\int_{0}^{\tau} \int_{\Omega} F \nabla\left(\operatorname{sign} u(|u|-k)_{+}\right) \mathrm{d} x \mathrm{~d} t .
\end{aligned}
$$

For the first term of (3.10), by Lemma 3.2 (or Corollary 3.1) we have

$$
\left\langle\frac{\partial u}{\partial t}, \operatorname{sign} u(|u|-k)_{+}\right\rangle=\frac{1}{2} \int_{\Omega}\left[(|u(x, \tau)|-k)_{+}\right]^{2} \mathrm{~d} x .
$$

Set

$$
A_{k}(t)=\{x \in \Omega:|u(x, t)|>k\}, \quad \psi(k)=\int_{0}^{T} \operatorname{meas} A_{k}(t) \mathrm{d} t .
$$

Concerning the second term of (3.10), we estimate as follows:

$$
\begin{gathered}
\int_{0}^{\tau} \int_{\Omega} a(x, t, u, \nabla u) \nabla\left(\operatorname{sign} u(|u|-k)_{+}\right) \mathrm{d} x \mathrm{~d} t \\
\quad \geq \alpha \int_{0}^{\tau} \int_{A_{k}(t)}\left|\nabla(|u|-k)_{+}\right|^{p(x, t)} \mathrm{d} x \mathrm{~d} t .
\end{gathered}
$$

Since $1<p^{-} \leq p(x, t)$, applying Hölder's inequality we have

$$
\begin{aligned}
& \int_{0}^{\tau} \int_{A_{k}(t)}|\nabla u|^{p^{-}} \mathrm{d} x \mathrm{~d} t \\
& \quad \leq \int_{0}^{\tau} \int_{A_{k}(t)}|\nabla u|^{p(x, t)} \mathrm{d} x \mathrm{~d} t+\int_{A_{k}(t)}|\nabla u| \mathrm{d} x \mathrm{~d} t \\
& \quad \leq \int_{0}^{\tau} \int_{A_{k}(t)}|\nabla u|^{p(x, t)} \mathrm{d} x \mathrm{~d} t+\left(\int_{0}^{\tau} \int_{A_{k}(t)}|\nabla u|^{p^{-}} \mathrm{d} x \mathrm{~d} t\right)^{\frac{1}{p^{-}}} \psi(k)^{\frac{p^{-}-1}{p^{-}}} \\
& \quad \leq \int_{0}^{\tau} \int_{A_{k}(t)}|\nabla u|^{p(x, t)} \mathrm{d} x \mathrm{~d} t+\left(\int_{0}^{\tau} \int_{A_{k}(t)}|\nabla u|^{p^{-}} \mathrm{d} x \mathrm{~d} t\right)^{\frac{1}{p^{-}}} \psi(k)^{\frac{p^{-}-1}{p^{-}}} .
\end{aligned}
$$


The above inequality and Young's inequality show that

$$
\frac{p^{-}-1}{p^{-}} \int_{0}^{\tau} \int_{A_{k}(t)}|\nabla u|^{p^{-}} \mathrm{d} x \mathrm{~d} t \leq \int_{0}^{\tau} \int_{A_{k}(t)}|\nabla u|^{p(x, t)} \mathrm{d} x \mathrm{~d} t+\frac{p^{-}-1}{p^{-}} \psi(k) .
$$

By $\left(\mathrm{H}_{5}\right)$, we may assume that $\phi=\left(\phi_{1}, \phi_{2}, \ldots, \phi_{N}\right)$, where $\phi_{i} \in C(\mathbb{R})$ for $1 \leq i \leq N$. Let $\tilde{\phi}_{i}(\theta)=$ $\int_{0}^{\theta} \chi_{\{|\eta| \geq k\}} \phi_{i}(\eta) \mathrm{d} \eta$ and set $\tilde{\phi}=\left(\tilde{\phi}_{1}, \tilde{\phi}_{2}, \ldots, \tilde{\phi}_{N}\right)$, then it is easy to see that, after using the divergence theorem,

$$
\begin{aligned}
\int_{0}^{\tau} \int_{\Omega} \phi(u) \nabla\left(\operatorname{sign} u(|u|-k)_{+}\right) \mathrm{d} x \mathrm{~d} t & =\int_{0}^{\tau} \int_{\Omega} \chi_{\{|u| \geq k\}} \phi(u) \cdot \nabla u \mathrm{~d} x \mathrm{~d} t \\
& =\int_{0}^{\tau} \int_{\Omega} \operatorname{div} \tilde{\phi}(u) \mathrm{d} x \mathrm{~d} t \\
& =\int_{0}^{\tau} \int_{\partial \Omega} \tilde{\phi}(u) \cdot \vec{n} \mathrm{~d} S \mathrm{~d} t=0
\end{aligned}
$$

where $\vec{n}$ is the outward pointing unit normal field of the boundary $\partial \Omega$.

Recalling that $\bar{M}-1 \leq k \leq \bar{M}$, it is straightforward that

$$
\left|\int_{0}^{\tau} \int_{\Omega} f \operatorname{sign} u(|u|-k)_{+} \mathrm{d} x \mathrm{~d} t\right| \leq \int_{0}^{\tau} \int_{A_{k}(t)}|f| \mathrm{d} x \mathrm{~d} t \leq\|f\|_{L^{q^{-}}(Q)} \psi(k)^{1-\frac{1}{q^{-}}} .
$$

For the second term of the right hand side of (3.10), we have

$$
\begin{aligned}
& \left|\int_{0}^{\tau} \int_{\Omega} F \nabla\left(\operatorname{sign} u(|u|-k)_{+}\right) \mathrm{d} x \mathrm{~d} t\right| \\
& \quad \leq C\left(\alpha, p^{-}\right) \int_{0}^{\tau} \int_{A(k)}|F|^{\frac{p^{-}}{p^{-1}}} \mathrm{~d} x \mathrm{~d} t+\frac{\alpha\left(p^{-}-1\right)}{2 p^{-}} \int_{0}^{\tau} \int_{A(k)}|\nabla u|^{p^{-}} \mathrm{d} x \mathrm{~d} t .
\end{aligned}
$$

It follows from (3.10)-(3.16) that

$$
\begin{aligned}
& \frac{1}{2} \int_{\Omega}\left[(|u|-k)_{+}\right]^{2}(\tau) \mathrm{d} x+\frac{\alpha\left(p^{-}-1\right)}{2 p^{-}} \int_{0}^{\tau} \int_{A_{k}(t)}\left|\nabla(|u|-k)_{+}\right|^{p^{-}} \mathrm{d} x \mathrm{~d} t \\
& \quad \leq C\left(\alpha, p^{-}\right)\left[\left(\int_{0}^{\tau} \int_{A(k)}|F|^{\frac{q^{-} p^{-}}{p^{-}-1}} \mathrm{~d} x \mathrm{~d} t\right)^{\frac{1}{q^{-}}}+\|f\|_{L^{q^{-}}(Q)}\right] \psi(k)^{1-\frac{1}{q^{-}}}+\frac{p^{-}-1}{p^{-}} \psi(k) .
\end{aligned}
$$

Taking the supremum for $\tau \in[0, T]$, we obtain

$$
\begin{aligned}
& \sup _{0 \leq \tau \leq T} \int_{\Omega}\left[(|u|-k)_{+}\right]^{2}(\tau) \mathrm{d} x+\frac{\alpha\left(p^{-}-1\right)}{p^{-}} \int_{0}^{T} \int_{A_{k}(t)}\left|\nabla(|u|-k)_{+}\right|^{p^{-}} \mathrm{d} x \mathrm{~d} t \\
& \quad \leq C\left(\alpha, p^{-}, N,\|f\|_{L^{q^{-}}(Q)},\left\||F|^{\frac{p^{-}}{p^{-}-1}}\right\|_{L^{q^{-}}(Q)}\right) \psi(k)^{1-\frac{1}{q^{-}}}+2 \frac{p^{-}-1}{p^{-}} \psi(k) .
\end{aligned}
$$

Now considering the sequence

$$
k_{n}=\bar{M}-\varepsilon-\frac{\varepsilon}{2^{n}}, \quad \text { for } n=0,1,2 \ldots,
$$


replacing $k$ by $k_{n}$ in (3.17) and using Lemma 3.1 of [18], we find that

$$
\begin{aligned}
&\left(\frac{\varepsilon}{2^{n+1}}\right)^{\frac{p^{-}(N+2)}{N}} \psi\left(k_{n+1}\right) \\
& \leq \int_{Q}\left|\left(|u|-k_{n}\right)_{+}\right|^{\frac{p^{-}(N+2)}{N}} \mathrm{~d} x \mathrm{~d} t \\
& \leq C\left(p^{-}, N\right)\left(\sup _{0 \leq \tau \leq T} \int_{\Omega}\left[\left(|u|-k_{n}\right)_{+}\right]^{2}(\tau) \mathrm{d} x\right)^{\frac{p^{-}}{N}} \int_{0}^{T} \int_{A_{k}(t)}\left|\nabla\left(|u|-k_{n}\right)_{+}\right|^{p^{-}} \mathrm{d} x \mathrm{~d} t \\
& \leq C\left(\alpha, p^{-}, N,\|f\|_{L^{q^{-}}(Q)},\left\||F|^{\frac{p^{-}}{p^{-1}}}\right\|_{L^{q^{-}}(Q)}\right) \\
& \times\left[\psi\left(k_{n}\right)^{1-\frac{1}{q^{-}}}+\psi\left(k_{n}\right)\right]\left[\psi\left(k_{n}\right)^{\left(1-\frac{1}{q^{-}}\right) \frac{p^{-}}{N}}+\psi\left(k_{n}\right)^{\frac{p^{-}}{N}}\right] \\
& \leq C_{0}\left[\psi\left(k_{n}\right)^{\beta_{1}}+\psi\left(k_{n}\right)^{\beta_{2}}+\psi\left(k_{n}\right)^{\beta_{3}}+\psi\left(k_{n}\right)^{\beta_{4}}\right],
\end{aligned}
$$

where $C_{0}:=C\left(\alpha, p^{-}, N,\|f\|_{L^{q^{-}}(Q)},\left\||F|^{\frac{p^{-}}{p^{-1}}}\right\|_{L^{q^{-}}(Q)}\right)$ and

$$
\begin{aligned}
& \beta_{1}=\left(1-\frac{1}{q^{-}}\right)\left(1+\frac{p^{-}}{N}\right), \quad \beta_{2}=1-\frac{1}{q^{-}}+\frac{p^{-}}{N}, \\
& \beta_{3}=1+\left(1-\frac{1}{q^{-}}\right) \frac{p^{-}}{N}, \quad \beta_{4}=1+\frac{p^{-}}{N} .
\end{aligned}
$$

The equality (3.18) is equivalent to

$$
\psi\left(k_{n+1}\right) \leq C_{0} 2^{2 p} b^{n} \varepsilon \frac{-p^{-}(N+2)}{N}\left[\psi\left(k_{n}\right)^{\beta_{1}}+\psi\left(k_{n}\right)^{\beta_{2}}+\psi\left(k_{n}\right)^{\beta_{3}}+\psi\left(k_{n}\right)^{\beta_{4}}\right],
$$

where $b=2^{\frac{p^{-}(N+2)}{N}}$.

It follows from Lemma 3.4 that

$$
\lim _{n \rightarrow \infty} \psi\left(k_{n}\right)=0
$$

provided

$$
\psi\left(k_{0}\right) \leq c^{*} \equiv\left(\frac{\varepsilon^{\frac{p^{-}(N+2)}{N}}}{C_{0} 2^{2(p+1)}}\right)^{\frac{1}{\beta-1}} b^{\frac{-1}{(\beta-1)^{2}}}
$$

here $\beta=\min _{1 \leq i \leq 4}\left\{\beta_{i}\right\}$.

In view of (3.20), we arrive that

$$
|u| \leq \bar{M}-\varepsilon,
$$

which contradicts the definition of $\bar{M}$.

Since

$$
\left(\frac{\bar{M}}{2}\right)^{p^{-}} \psi\left(k_{0}\right) \leq\left(\frac{\bar{M}}{2}\right)^{p^{-}} \psi\left(\frac{\bar{M}}{2}\right) \leq \int_{0}^{T} \int_{\Omega}|u|^{p^{-}} \mathrm{d} x \mathrm{~d} t
$$


i.e.

$$
\psi\left(\frac{\bar{M}}{2}\right) \leq\left(\frac{2}{\bar{M}}\right)^{p^{-}} \int_{0}^{T} \int_{\Omega}|u|^{p^{-}} \mathrm{d} x \mathrm{~d} t
$$

we have a contradiction of (3.21) if $\left(\frac{2}{M}\right)^{p^{-}} \int_{0}^{T} \int_{\Omega}|u|^{p^{-}} \mathrm{d} x \mathrm{~d} t \leq c^{*}$. Hence, we conclude that

$$
\bar{M} \leq 2\left(c^{*}\right)^{-\frac{1}{p^{-}}}\left(\int_{0}^{T} \int_{\Omega}|u|^{p^{-}} \mathrm{d} x \mathrm{~d} t\right)^{\frac{1}{p^{-}}}
$$

Now taking $\eta(x, t)=u$ as a test function in problem $(P)$ and arguing as in (3.13), we find that

$$
\begin{aligned}
& \int_{\Omega} u^{2}(\tau) \mathrm{d} x+\frac{\alpha\left(p^{-}-1\right)}{p^{-}} \int_{0}^{\tau} \int_{\Omega}|\nabla u|^{p^{-}} \mathrm{d} x \mathrm{~d} t \\
& \leq C\left(\alpha, p^{-}\right) \int_{0}^{\tau} \int_{\Omega}|F|^{\frac{p^{-}}{p^{-}}} \mathrm{d} x \mathrm{~d} t+\frac{\alpha\left(p^{-}-1\right)}{2 p^{-}} \int_{0}^{\tau} \int_{\Omega}|\nabla u|^{p^{-}} \mathrm{d} x \mathrm{~d} t \\
& \quad+\bar{M} \int_{0}^{\tau} \int_{\Omega}|f| \mathrm{d} x \mathrm{~d} t+\left\|u_{0}\right\|_{L^{\infty}(\Omega)}^{2},
\end{aligned}
$$

where we have used similar results to (3.14) and (3.16).

Taking the supremum for $\tau \in[0, T]$, we have

$$
\begin{aligned}
& \sup _{0 \leq \tau \leq T} \int_{\Omega} u^{2}(\tau) \mathrm{d} x+\frac{\alpha\left(p^{-}-1\right)}{2 p^{-}} \int_{0}^{T} \int_{\Omega}|\nabla u|^{p^{-}} \mathrm{d} x \mathrm{~d} t \\
& \leq C\left(\alpha, p^{-}\right) \int_{0}^{T} \int_{\Omega}|F|^{\frac{p^{-}}{p^{-1}}} \mathrm{~d} x \mathrm{~d} t+\bar{M} \int_{0}^{T} \int_{\Omega}|f| \mathrm{d} x \mathrm{~d} t+\left\|u_{0}\right\|_{L^{\infty}(\Omega)}^{2} \\
& \leq C\left(\alpha, p^{-}\right) \int_{0}^{T} \int_{\Omega}|F|^{\frac{p^{-}}{p^{-}}} \mathrm{d} x \mathrm{~d} t \\
& \quad+2 C(\Omega, N)\left(c^{*}\right)^{-\frac{1}{p^{-}}}\|\nabla u\|_{L^{p^{-}}(Q)} \int_{0}^{T} \int_{\Omega}|f| \mathrm{d} x \mathrm{~d} t+\left\|u_{0}\right\|_{L^{\infty}(\Omega)}^{2},
\end{aligned}
$$

where we have used the Poincaré inequality, (3.22), and (3.23).

Applying Young's inequality and the Poincaré inequality in (3.24), we obtain

$$
\left(\int_{0}^{T} \int_{\Omega}|u|^{p^{-}} \mathrm{d} x \mathrm{~d} t\right)^{\frac{1}{p^{-}}} \leq C\left(\alpha, p^{-}, \Omega, N,\|f\|_{L^{q^{-}}(Q)},\left\||F|^{\frac{p^{-}}{p^{-1}}}\right\|_{L^{q^{-}}(Q)},\left\|u_{0}\right\|_{L^{\infty}(\Omega)}\right) .
$$

From (3.22) and (3.25), we obtain the desired result of Lemma 3.3.

To prove Theorem 3.1, we have to consider approximating problems. We define a truncation $\bar{a}$ of $a$ by

$$
\bar{a}(x, t, s, \xi)=a\left(x, t, T_{M}(s), \xi\right), \quad \text { a.e. }(x, t) \in Q, \forall s \in \mathbb{R} \text { and } \xi \in \mathbb{R}^{N},
$$

where $M$ is defined as in Lemma 3.3. 
Similarly, the truncation $\bar{\phi}$ of $\phi$ is defined as

$$
\bar{\phi}(s)=\phi\left(T_{M}(s)\right), \quad \forall s \in \mathbb{R} .
$$

For each $\varepsilon>0$, we define

$$
g_{\varepsilon}(x, t, s)=\frac{g(x, t, s)}{1+\varepsilon|g(x, t, s)|}, \quad \text { a.e. }(x, t) \in Q, \forall s \in \mathbb{R},
$$

and the sequences $\left\{f_{\varepsilon}\right\} \subseteq C_{c}^{\infty}(\Omega)$ and $\left\{F_{\varepsilon}\right\} \subseteq C_{c}^{\infty}(\Omega)$ such that

$$
f_{\varepsilon} \rightarrow f \quad \text { strongly in } L^{q(x, t)}(\Omega), \quad F_{\varepsilon} \rightarrow F \quad \text { strongly in } F \in\left(L^{q(x, t)\left(p^{-}\right)^{\prime}}(Q)\right)^{N} .
$$

Obviously, the function $\bar{a}$ satisfies $\left(\mathrm{H}_{1}\right),\left(\mathrm{H}_{3}\right)$ with $a$ replaced by $\bar{a}$. Moreover, due to $\left(\mathrm{H}_{2}\right)$, there exist $\bar{c} \in L^{p^{\prime}(\cdot)}(\Omega)$ and a constant $b_{M}>0$ such that

$$
|\bar{a}(x, t, s, \xi)| \leq b_{M}|\xi|^{p(x, t)-1}+\bar{c}(x, t), \quad \text { a.e. }(x, t) \in Q, \forall s \in \mathbb{R} \text { and } \forall \xi \in \mathbb{R}^{N}
$$

Now, we introduce a family of approximate problems:

$$
\left(P_{\varepsilon}\right) \begin{cases}\frac{\partial u_{\varepsilon}}{\partial t}-\operatorname{div} \bar{a}\left(x, t, u_{\varepsilon}, \nabla u_{\varepsilon}\right)-\operatorname{div} \bar{\phi}\left(u_{\varepsilon}\right)+g_{\varepsilon}\left(x, t, u_{\varepsilon}\right)=f_{\varepsilon}-\operatorname{div} F_{\varepsilon} & \text { in } Q, \\ u_{\varepsilon}=0 & \text { on } \partial \Omega \times(0, T), \\ u_{\varepsilon}(x, 0)=u_{0} & \text { in } \Omega .\end{cases}
$$

In the following, we prove the existence of weak solutions of problem $\left(P_{\varepsilon}\right)$. We will solve problem $\left(P_{\varepsilon}\right)$ by Galerkin's method.

For every fixed $t \in[0, T]$, we introduce the Banach space

$$
V_{t}(\Omega)=\left\{u \text { is measurable }: u \in L^{2}(\Omega) \cap W_{0}^{1, p(x, t)}(\Omega)\right\}
$$

endowed with the norm

$$
\|u\|_{V_{t}(\Omega)}:=\|u\|_{L^{2}(\Omega)}+\|\nabla u\|_{L^{p(x, t)}(\Omega)}
$$

It is easy to see that $V_{t}(\Omega)$ is reflexive and separable as a closed subspace of $W_{0}^{1, p^{-}}(\Omega) \cap$ $L^{2}(\Omega)$. Hence there exists a countable set of linearly independent functions $\left\{\varphi_{i}\right\}_{i=1}^{\infty} \subseteq$ $C_{0}^{\infty}(\Omega)$ consists a basis of $V_{t}(\Omega)$. Without loss of generality, we may assume that $\left\{\varphi_{i}\right\}_{i=1}^{\infty}$ also forms an orthonormal basis of $L^{2}(\Omega)$. Fix now a positive integer $m$ and let

$$
V_{m}=\operatorname{span}\left\{\varphi_{1}, \ldots, \varphi_{m}\right\}
$$

One can check that, for any given $v \in W(Q) \cap L^{2}(Q)$, there is a sequence $v_{m}^{i}(t) \in C^{1}[0, T]$ such that

$$
v_{m}=\sum_{i=1}^{m} v_{m}^{i}(t) \varphi_{i} \rightarrow v \quad \text { strongly in } W(Q) \cap L^{2}(Q) .
$$


Now we consider the following approximate problem: find

$$
u_{m}(t)=\sum_{i=1}^{m} u_{m}^{i}(t) \varphi_{i}
$$

where the coefficients $u_{m}^{i}(t)$ satisfies

$$
\begin{aligned}
& \int_{\Omega} \frac{\partial u_{m}}{\partial t} \varphi_{i} \mathrm{~d} x+\int_{\Omega}\left[\bar{a}\left(x, t, u_{m}, \nabla u_{m}\right)+\bar{\phi}\left(u_{m}\right)\right] \cdot \nabla \varphi_{i} \mathrm{~d} x+\int_{\Omega} g_{\varepsilon}\left(x, t, u_{m}\right) \varphi_{i} \mathrm{~d} x \\
&=\int_{\Omega}\left[f_{\varepsilon}-\operatorname{div} F_{\varepsilon}\right] \varphi_{i} \mathrm{~d} x,
\end{aligned}
$$

for $i=1,2, \ldots, m$ and $0 \leq t \leq T$, where

$$
u_{m}^{i}(0)=\int_{\Omega} u_{0}(x) \varphi_{i} \mathrm{~d} x, \quad i=1,2, \ldots, m .
$$

The existence result of problem (3.28) and (3.29) is stated as follows.

Lemma 3.5 Fixed $\varepsilon>0$, for each positive integer $m=1,2, \ldots$, there exists a function $u_{m}$ of the form (3.28) satisfying (3.29).

Proof of Lemma 3.5 In order to prove our results, we introduce the following notations. For any element of $v \in V$, we denote by

$$
\vec{v}_{m}=\left\{v^{1}, v^{2}, \ldots, v^{m}\right\} \in \mathbb{R}^{m}
$$

associated with the projection $v^{m}$ of $v$ on $V_{m}$, i.e. $v^{i} \in \mathbb{R}$ such that

$$
v_{m}=\sum_{i=1}^{m} v^{i} \varphi_{i} \rightarrow v \quad \text { strongly in } V, \text { as } m \rightarrow+\infty .
$$

Let $G^{m}$ be the mapping from $\mathbb{R}^{m}$ into itself whose $i$ th component is

$$
\left[G^{m}\left(\vec{v}_{m}\right)\right]_{i}=\int_{\Omega}\left[\bar{a}\left(x, t, v_{m}, \nabla v_{m}\right)+\bar{\phi}\left(v_{m}\right)\right] \cdot \nabla \varphi_{i} \mathrm{~d} x+\int_{\Omega} g_{\varepsilon}\left(x, t, v_{m}\right) \varphi_{i} \mathrm{~d} x,
$$

respectively.

Also we define $F^{m}(t)$ to be the vector of $\mathbb{R}^{m}$ whose $i$ th component is

$$
\left[F^{m}(t)\right]_{i}=\int_{\Omega}\left(f_{\varepsilon}(x, t)-\operatorname{div} F_{\varepsilon}(x, t)\right) \varphi_{i} \mathrm{~d} x .
$$

With the above notations, the problem (3.28) and (3.29) can be written as follows:

$$
\frac{d}{d t} \vec{u}_{m}(t)+G^{m}\left(\vec{u}_{m}(t)\right)=F^{m}(t), \quad \vec{u}_{m}(0)=\left\{u_{m}^{1}(0), u_{m}^{2}(0), \ldots, u_{m}^{m}(0)\right\} .
$$

It is easy to check that $G^{m}$ and $F^{m}(t)$ are continuous. Hence, the ordinary system (3.30) has a local $C^{1}$ solution $\vec{u}_{m}(t)$ on some interval $\left[0, t_{m}\right]$, where $t_{m}$ is a positive number. 
Now we prove that $t_{m}=T$. We still need some $a$ priori estimates for the sequence of $\left\{u_{m}\right\}$.

Multiplication of the first equality of (3.30) by $\vec{u}_{m}(t)$ and integration over $(0, t)$, we obtain

$$
\begin{aligned}
& \frac{1}{2} \| u_{m}(t) \|_{L^{2}(\Omega)}^{2}+\int_{0}^{t} \int_{\Omega} \bar{a}\left(x, t, u_{m}, \nabla u_{m}\right) \nabla u_{m} \mathrm{~d} x \mathrm{~d} t+\int_{0}^{t} \int_{\Omega} \bar{\phi}\left(u_{m}\right) \nabla u_{m} \mathrm{~d} x \mathrm{~d} t \\
& \quad+\int_{0}^{t} \int_{\Omega} g_{\varepsilon}\left(x, t, u_{m}\right) u_{m} \mathrm{~d} x \mathrm{~d} t \\
&=\int_{0}^{t} \int_{\Omega}\left(f_{\varepsilon}(x, t)+\operatorname{div} F_{\varepsilon}(x, t)\right) u_{m} \mathrm{~d} x \mathrm{~d} t+\frac{1}{2}\left\|u_{m}(0)\right\|_{L^{2}(\Omega)}^{2} .
\end{aligned}
$$

Note that $\int_{0}^{t} \int_{\Omega} \bar{\phi}\left(u_{m}\right) \nabla u_{m} \mathrm{~d} x \mathrm{~d} t=0$, by Young's inequality, assumption $\left(\mathrm{H}_{1}\right)$ and $\left(\mathrm{H}_{4}\right)$ we get

$$
\frac{1}{2}\left\|u_{m}(t)\right\|_{L^{2}(\Omega)}^{2}+\alpha \int_{0}^{t} \int_{\Omega}\left|\nabla u_{m}\right|^{p(x, t)} \mathrm{d} x \mathrm{~d} t \leq C
$$

which implies that $\vec{u}_{m}(t)$ remains bounded as $t$ tends to $T_{m}$, where $C$ is a positive constant independent of $m$.

Since $\vec{u}_{m}(t)$ does not blow up whenever $t$ tends to $t_{m}$, the system (3.30) admits a global solution on $[0, T]$. Thus, we have finished the proof.

Proof of Theorem 3.1 The proof is divided into three steps.

Step 1: we prove the existence of solutions to problem $\left(P_{\varepsilon}\right)$.

In view of (3.32) and Lemma 2.1, we infer that the solution $u_{m}$ obtained in Lemma 3.5 is bounded in $W(Q) \cap L^{\infty}\left(0, T ; L^{2}(\Omega)\right)$ with respect to $m$. Hence, there exists a subsequence of $\left\{u_{m}\right\}$ (still denoted by $\left\{u_{m}\right\}$ ) such that as $m \rightarrow \infty$,

$$
\begin{aligned}
& \nabla u_{m} \rightarrow \nabla u_{\varepsilon} \quad \text { weakly in }\left(L^{p(x, t)}(Q)\right)^{N} \text { and weakly* in } L^{\infty}\left(0, T ; L^{2}(\Omega)\right), \\
& u_{m} \rightarrow u_{\varepsilon} \quad \text { weakly in } L^{p^{-}}\left(0, T ; W_{0}^{1, p(x, t)}(\Omega)\right),
\end{aligned}
$$

and

$$
\bar{a}\left(x, t, u_{m}, \nabla u_{m}\right) \rightarrow \zeta_{\varepsilon} \quad \text { weakly in }\left(L^{p^{\prime}(x, t)}(Q)\right)^{N} .
$$

Moreover, we have

$$
\begin{aligned}
& \frac{\partial u_{m}}{\partial t} \text { is bounded in } W^{\star}(Q) \text { with respect to } m, \\
& W_{0}^{1, p^{-}}(\Omega) \hookrightarrow \hookrightarrow L^{s}(\Omega) \subseteq\left(W_{0}^{1, \lambda}(\Omega)\right)^{\star}, \quad \text { for } 1 \leq s<\frac{N p^{-}}{N-p^{-}} \text {and } \lambda>\left\{\frac{N s}{N(s-1)+s}, p^{+}\right\} .
\end{aligned}
$$

By the above results, Lemma 3.1, it is easy to see that

$$
\begin{aligned}
& \frac{\partial u_{m}}{\partial t} \text { is bounded in } L^{\left(p^{+}\right)^{\prime}}\left(0, T ;\left(W_{0}^{1, \lambda}(\Omega)\right)^{\star}\right) \text { and } \\
& u_{m} \text { is bounded in } L^{p^{-}}\left(0, T ; W_{0}^{1, p^{-}}(\Omega)\right) .
\end{aligned}
$$


Then using an Aubin's type lemma (see Corollary 4 of [26]), we conclude that $\left\{u_{m}\right\}$ contains a subsequence strongly convergent in $L^{\tilde{s}}(Q)$, where $\tilde{s}=\min \left\{p^{-}, s\right\}$. Thus, we can also draw a subsequence of $\left\{u_{m}\right\}$ (still denoted by $\left\{u_{m}\right\}$ ) such that

$$
u_{m} \rightarrow u_{\varepsilon} \quad \text { a.e. in } Q \text {. }
$$

Since $u_{m}$ satisfies (3.28) and (3.29), it is easy to see that for all $\varphi \in C^{1}\left([0, T] ; V_{m}\right)$ and $\tau \in$ $(0, T]$,

$$
\begin{gathered}
\int_{0}^{\tau} \int_{\Omega} \frac{\partial u_{m}}{\partial t} \varphi \mathrm{d} x \mathrm{~d} t+\int_{0}^{\tau} \int_{\Omega}\left[\bar{a}\left(x, t, u_{m}, \nabla u_{m}\right)+\bar{\phi}\left(u_{m}\right)\right] \cdot \nabla \varphi \mathrm{d} x \mathrm{~d} t \\
\quad+\int_{0}^{\tau} \int_{\Omega} g_{\varepsilon}\left(x, t, u_{m}\right) \varphi \mathrm{d} x \mathrm{~d} t=\int_{0}^{\tau} \int_{\Omega}\left[f_{\varepsilon}-\operatorname{div} F_{\varepsilon}\right] \varphi \mathrm{d} x \mathrm{~d} t .
\end{gathered}
$$

Since, for any given $\varphi \in C^{1}\left(0, T ; C_{0}^{\infty}(\Omega)\right)$, there exists a sequence $\varphi_{m} \in C^{1}\left([0, T] ; V_{m}\right)$ such that $\varphi_{m} \rightarrow \varphi$ in $C^{1}\left(0, T ; C_{0}^{\infty}(\Omega)\right)$, we have

$$
\lim _{m \rightarrow \infty} \int_{0}^{\tau} \int_{\Omega} \frac{\partial u_{m}}{\partial t} \varphi \mathrm{d} x \mathrm{~d} t=\lim _{m \rightarrow \infty} \int_{0}^{\tau} \int_{\Omega} \frac{\partial u_{m}}{\partial t} \varphi_{m} \mathrm{~d} x \mathrm{~d} t, \quad \forall \varphi \in C^{1}\left(0, T ; C_{0}^{\infty}(\Omega)\right) .
$$

As a consequence, it follows from (3.33)-(3.36) that

$$
\begin{aligned}
& \lim _{m \rightarrow \infty} \int_{0}^{\tau} \int_{\Omega} \frac{\partial u_{m}}{\partial t} \varphi \mathrm{d} x \mathrm{~d} t+\int_{0}^{\tau} \int_{\Omega}\left(\zeta_{\varepsilon}+\bar{\phi}\left(u_{\varepsilon}\right)\right) \cdot \nabla \varphi \mathrm{d} x \mathrm{~d} t+\int_{0}^{\tau} \int_{\Omega} g_{\varepsilon}\left(x, t, u_{\varepsilon}\right) \varphi \mathrm{d} x \mathrm{~d} t \\
& =\int_{0}^{\tau} \int_{\Omega}\left[f_{\varepsilon}-\operatorname{div} F_{\varepsilon}\right] \varphi \mathrm{d} x \mathrm{~d} t, \quad \forall \varphi \in C^{1}\left(0, T ; C_{0}^{\infty}(\Omega)\right), \tau \in(0, T] .
\end{aligned}
$$

To identity the term $\zeta_{\varepsilon}$, we shall prove the following result:

$$
\varlimsup_{m \rightarrow \infty} \int_{0}^{T} \int_{0}^{\tau} \int_{\Omega} \bar{a}\left(x, t, u_{m}, \nabla u_{m}\right) \nabla u_{m} \mathrm{~d} x \mathrm{~d} t \mathrm{~d} \tau \leq \int_{0}^{T} \int_{0}^{\tau} \int_{\Omega} \zeta_{\varepsilon} \nabla u_{\varepsilon} \mathrm{d} x \mathrm{~d} t \mathrm{~d} \tau .
$$

For this purpose, we need to choose an appropriate test function $\varphi$ in (3.38). We will use the regularization method of Landes [27]. We define the regularization in time of the function $u_{\varepsilon}$ by

$$
\left(u_{\varepsilon}\right)_{v}(x, t)=v \int_{-\infty}^{t} e^{v(\theta-t)} \bar{u}_{\varepsilon}(x, \theta) \mathrm{d} \theta \quad \text { for } v \in \mathbb{N}
$$

where $\bar{u}_{\varepsilon}(x, \theta)=u_{\varepsilon}(x, \theta)$ if $\theta>0 ; \bar{u}_{\varepsilon}(x, \theta)=0$ if $\theta \leq 0$.

As in [27], the function $\left(u_{\varepsilon}\right)_{v} \in W^{1, p^{-}}\left(0, T ; W_{0}^{1, p(x, t)}(\Omega)\right) \cap W(Q) \cap L^{2}(Q)$ satisfies

$$
\frac{\partial}{\partial t}\left(u_{\varepsilon}\right)_{v}+v\left(\left(u_{\varepsilon}\right)_{v}-u_{\varepsilon}\right)=0
$$

and

$$
\left(u_{\varepsilon}\right)_{v} \rightarrow u_{\varepsilon} \quad \text { a.e. in } Q \text { and strongly in } W(Q) .
$$


In order to deal with a nonzero initial datum $u_{0}$, we now define

$$
\left(u_{\varepsilon}\right)_{v, j}=\left(u_{\varepsilon}\right)_{v}(x, t)+e^{-v t} u_{0 j}
$$

where $\left\{u_{0 j}\right\} \subseteq C_{0}^{\infty}(\Omega)$ such that $u_{0 j} \rightarrow u_{0}$ strongly in $L^{\sigma}(\Omega)$ (for any $\sigma \geq 1$ ) and weakly* in $L^{\infty}(\Omega)$.

Obviously, this function satisfies the following problems:

$$
\left\{\begin{array}{l}
\frac{\partial}{\partial t}\left(u_{\varepsilon}\right)_{v, j}+v\left(\left(u_{\varepsilon}\right)_{v, j}-u_{\varepsilon}\right)=0 \\
\left.\left(u_{\varepsilon}\right)_{v, j}\right|_{t=0}=u_{0 j}
\end{array}\right.
$$

Moreover, the $\left(u_{\varepsilon}\right)_{v, j} \in W^{1, p^{-}}\left(0, T ; W_{0}^{1, p(x, t)}(\Omega)\right) \cap W(Q) \cap L^{2}(Q)$ enjoys the property

$$
\left(u_{\varepsilon}\right)_{v, j} \rightarrow u_{\varepsilon} \quad \text { a.e. in } Q \text { and strongly in } W(Q) .
$$

Choosing $\varphi=\left(u_{\varepsilon}\right)_{v, j}$ as a test function in (3.38) and taking $\varphi=u_{m}$ in (3.37), we get

$$
\begin{aligned}
\varlimsup_{j \rightarrow \infty} & \varlimsup_{\nu \rightarrow \infty} \varlimsup_{m \rightarrow \infty} I(m, v, j) \\
\leq & \varlimsup_{j \rightarrow \infty} \varlimsup_{v \rightarrow \infty} \varlimsup_{m \rightarrow \infty} I_{1}(m, v, j)+\varlimsup_{j \rightarrow \infty} \varlimsup_{\nu \rightarrow \infty} \varlimsup_{m \rightarrow \infty} I_{2}(m, v, j) \\
& +\varlimsup_{j \rightarrow \infty} \varlimsup_{v \rightarrow \infty} \varlimsup_{m \rightarrow \infty} I_{3}(m, v, j)+\varlimsup_{j \rightarrow \infty} \varlimsup_{v \rightarrow \infty} \varlimsup_{m \rightarrow \infty} I_{4}(m, v, j),
\end{aligned}
$$

where

$$
\begin{aligned}
& I(m, v, j)=\int_{0}^{T} \int_{0}^{\tau} \int_{\Omega}\left[\bar{a}\left(x, t, u_{m}, \nabla u_{m}\right) \nabla u_{m}-\zeta_{\varepsilon} \nabla\left(u_{\varepsilon}\right)_{v, j}\right] \mathrm{d} x \mathrm{~d} t \mathrm{~d} \tau \\
& I_{1}(m, v, j)=-\int_{0}^{T} \int_{0}^{\tau} \int_{\Omega} \frac{\partial u_{m}}{\partial t}\left[u_{m}-\left(u_{\varepsilon}\right)_{v, j}\right] \mathrm{d} x \mathrm{~d} t \mathrm{~d} \tau \\
& I_{2}(m, v, j)=-\int_{0}^{T} \int_{0}^{\tau} \int_{\Omega} \bar{\phi}\left(u_{m}\right) \nabla\left(u_{m}-\left(u_{\varepsilon}\right)_{v, j}\right) \mathrm{d} x \mathrm{~d} t \mathrm{~d} \tau \\
& I_{3}(m, v, j)=\int_{0}^{T} \int_{0}^{\tau} \int_{\Omega}\left[u_{m}-\left(u_{\varepsilon}\right)_{v, j}\right]\left[f_{\varepsilon}-\operatorname{div} F_{\varepsilon}\right] \mathrm{d} x \mathrm{~d} t \mathrm{~d} \tau, \\
& I_{4}(m, v, j)=\int_{0}^{T} \int_{0}^{\tau} \int_{\Omega} g_{\varepsilon}\left(x, t, u_{m}\right)\left[u_{m}-\left(u_{\varepsilon}\right)_{v, j}\right] \mathrm{d} x \mathrm{~d} t \mathrm{~d} \tau .
\end{aligned}
$$

In the following, we pass to the limit in (3.41) as $m \rightarrow \infty, v \rightarrow \infty$, and then $j \rightarrow \infty$.

The limit of $I_{1}(m, v, j)$ : we rewrite $I_{1}(m, v, j)$ as follows:

$$
\begin{aligned}
I_{1}(m, v, j)= & -\int_{0}^{T} \int_{0}^{\tau} \int_{\Omega}\left[\frac{\partial u_{m}}{\partial t}-\frac{\partial\left(u_{\varepsilon}\right)_{v, j}}{\partial t}\right]\left[u_{m}-\left(u_{\varepsilon}\right)_{v, j}\right] \mathrm{d} x \mathrm{~d} t \mathrm{~d} \tau \\
& -\int_{0}^{T} \int_{0}^{\tau} \int_{\Omega} \frac{\partial\left(u_{\varepsilon}\right)_{v, j}}{\partial t}\left[u_{m}-\left(u_{\varepsilon}\right)_{v, j}\right] \mathrm{d} x \mathrm{~d} t \mathrm{~d} \tau \\
= & I_{11}+I_{12} .
\end{aligned}
$$


For $I_{11}$, we have

$$
\begin{aligned}
I_{11} & =-\int_{0}^{T} \int_{0}^{\tau} \int_{\Omega}\left[\frac{\partial u_{m}}{\partial t}-\frac{\partial\left(u_{\varepsilon}\right)_{v, j}}{\partial t}\right]\left[u_{m}-\left(u_{\varepsilon}\right)_{\nu, j}\right] \mathrm{d} x \mathrm{~d} t \mathrm{~d} \tau \\
& =-\frac{1}{2} \int_{0}^{T} \int_{\Omega}\left[u_{m}-\left(u_{\varepsilon}\right)_{\nu, j}\right]^{2} \mathrm{~d} x \mathrm{~d} t+\frac{T}{2} \int_{\Omega}\left[u_{m}(x, 0)-u_{0 j}\right]^{2} \mathrm{~d} x \\
& \leq \frac{T}{2} \int_{\Omega}\left[u_{m}(x, 0)-u_{0 j}\right]^{2} \mathrm{~d} x,
\end{aligned}
$$

which yields

$$
\varlimsup_{j \rightarrow \infty} \varlimsup_{\nu \rightarrow \infty} \varlimsup_{m \rightarrow \infty} I_{11} \leq 0 .
$$

Using the properties of $\left(u_{\varepsilon}\right)_{v, j}$ and (3.36), we get the following estimate for $I_{12}$ :

$$
\begin{aligned}
\lim _{m \rightarrow \infty} I_{12} & =\lim _{m \rightarrow \infty} \int_{0}^{T} \int_{0}^{\tau} \int_{\Omega} v\left[\left(u_{\varepsilon}\right)_{\nu, j}-u_{\varepsilon}\right]\left[u_{m}-\left(u_{\varepsilon}\right)_{\nu, j}\right] \mathrm{d} x \mathrm{~d} t \mathrm{~d} \tau \\
& =v \int_{0}^{T} \int_{0}^{\tau} \int_{\Omega}\left[\left(u_{\varepsilon}\right)_{\nu, j}-u_{\varepsilon}\right]\left[u_{\varepsilon}-\left(u_{\varepsilon}\right)_{\nu, j}\right] \mathrm{d} x \mathrm{~d} t \mathrm{~d} \tau \leq 0 .
\end{aligned}
$$

Substituting (3.43) and (3.44) into (3.42),

$$
\varlimsup_{j \rightarrow \infty} \varlimsup_{\nu \rightarrow \infty} \varlimsup_{m \rightarrow \infty} I_{1}(m, v, j) \leq 0
$$

The limit of $I_{2}(m, v, j), I_{3}(m, v, j)$, and $I_{4}(m, v, j)$ : by (3.33), (3.34), and (3.36), it is easy to see that

$$
\begin{aligned}
& \lim _{v \rightarrow \infty} \lim _{m \rightarrow \infty} I_{2}(m, v, j)=0, \\
& \lim _{v \rightarrow \infty} \lim _{m \rightarrow \infty} I_{3}(m, v, j)=0, \\
& \lim _{v \rightarrow \infty} \lim _{m \rightarrow \infty} I_{4}(m, v, j)=0 .
\end{aligned}
$$

As a consequence of (3.45)-(3.48), we find that

$$
\begin{aligned}
\varlimsup_{m \rightarrow \infty} \int_{0}^{T} \int_{0}^{\tau} \int_{\Omega} \bar{a}\left(x, t, u_{m}, \nabla u_{m}\right) \nabla u_{m} & \leq \varlimsup_{j \rightarrow \infty} \varlimsup_{\nu \rightarrow \infty} \int_{0}^{T} \int_{0}^{\tau} \int_{\Omega} \zeta_{\varepsilon} \nabla\left(u_{\varepsilon}\right)_{\nu, j} \mathrm{~d} x \mathrm{~d} t \mathrm{~d} \tau \\
& =\int_{0}^{T} \int_{0}^{\tau} \int_{\Omega} \zeta_{\varepsilon} \nabla u_{\varepsilon} \mathrm{d} x \mathrm{~d} t \mathrm{~d} \tau,
\end{aligned}
$$

i.e. (3.39) holds true.

Step 2: In this step, we identify the quantities $\zeta_{\varepsilon}$, and prove that $u_{\varepsilon}$ is a weak solution of problem $\left(P_{\varepsilon}\right)$.

Equation (3.39) implies that, as $m$ tends to infinity,

$$
\varlimsup_{m \rightarrow \infty} \int_{0}^{T} \int_{0}^{\tau} \int_{\Omega}\left[\bar{a}\left(x, t, u_{m}, \nabla u_{m}\right)-\bar{a}\left(x, t, u_{m}, \nabla u_{\varepsilon}\right)\right]\left[\nabla u_{m}-\nabla u_{\varepsilon}\right] \mathrm{d} x \mathrm{~d} t \mathrm{~d} \tau \leq 0
$$


As a consequence of (3.49) and $\left(\mathrm{H}_{3}\right)$, we have, for any $0<\tau<T$,

$$
\begin{aligned}
& {\left[\bar{a}\left(x, t, u_{m}, \nabla u_{m}\right)-\bar{a}\left(x, t, u_{m}, \nabla u_{\varepsilon}\right)\right]\left[\nabla u_{m}-\nabla u_{\varepsilon}\right] \rightarrow 0} \\
& \quad \text { strongly in } L^{1}(\Omega \times[0, \tau]) .
\end{aligned}
$$

At the possible expense of extending the functions of $u_{m}, a(x, t, s, \xi), g(x, t, s), f$, and $F$ on a time interval $(0, \bar{T})$ with $\bar{T}>T$, in such a way such that all the assumptions $\left(\mathrm{H}_{1}\right)-\left(\mathrm{H}_{4}\right)$ hold true and $u_{m}$ is still a solution of problem (3.28) and (3.29) with $T$ in place of $\bar{T}$, we conclude that the previous convergence result (3.50) holds true in $L^{1}(Q)$, i.e.

$$
\left[\bar{a}\left(x, t, u_{m}, \nabla u_{m}\right)-\bar{a}\left(x, t, u_{m}, \nabla u_{\varepsilon}\right)\right]\left[\nabla u_{m}-\nabla u_{\varepsilon}\right] \rightarrow 0 \quad \text { strongly in } L^{1}(Q) .
$$

Using (3.33), (3.34), (3.36), (3.51), and arguing as in [28], we see that as $m \rightarrow \infty$,

$$
\nabla u_{m} \rightarrow \nabla u_{\varepsilon} \quad \text { strongly in }\left(L^{p(x, t)}(Q)\right)^{N} \text { and a.e. in } Q \text {. }
$$

By (3.52), using the Vitali convergence theorem, we get, as $m \rightarrow \infty$,

$$
\bar{a}\left(x, t, u_{m}, \nabla u_{m}\right) \rightarrow \zeta_{\varepsilon}=\bar{a}\left(x, t, u_{\varepsilon}, \nabla u_{\varepsilon}\right) \quad \text { strongly in }\left(L^{p^{\prime}(x, t)}(Q)\right)^{N}
$$

Moreover, it is easy to see that

$$
\operatorname{div} \bar{\phi}\left(u_{m}\right) \rightarrow \operatorname{div} \bar{\phi}\left(u_{\varepsilon}\right) \quad \text { strongly in } W^{\star}(Q)
$$

and

$$
g_{\varepsilon}\left(x, t, u_{m}\right) \rightarrow g_{\varepsilon}\left(x, t, u_{\varepsilon}\right) \quad \text { strongly in } L^{r}(Q), \forall r>1 .
$$

It follows from (3.53)-(3.55) and (3.37) that

$$
\frac{\partial u_{m}}{\partial t} \rightarrow \frac{\partial u_{\varepsilon}}{\partial t} \quad \text { strongly in } W^{\star}(Q) .
$$

Applying now the Aubin type lemma, by the fact that the sequence $\left\{u_{m}\right\}$ is bounded in $L^{\infty}\left(0, T ; L^{2}(\Omega)\right)$, we get for $s>\max \left\{\frac{2 N}{N+2}, p^{+}\right\}$

$$
u_{m} \rightarrow u_{\varepsilon} \quad \text { strongly in } C\left([0, T] ;\left(W_{0}^{1, s}(\Omega)\right)^{\star}\right)
$$

which implies that $\left.u_{\varepsilon}\right|_{t=0}=u_{0}$.

Recalling that $u_{\varepsilon} \in W(Q)$ and using the result (iv) of Lemma 3.1, we deduce that $u_{\varepsilon} \in$ $C\left([0, T] ; L^{2}(\Omega)\right)$. Combining this fact with the above convergence results and (3.38), we see that $u_{\varepsilon}$ is a weak solution of problem $\left(P_{\varepsilon}\right)$.

In the following, we prove that $u_{\varepsilon}$ belongs to $L^{\infty}(Q)$. Let $k$ be chosen so that $k \geq$ $\left\|u_{0}\right\|_{L^{\infty}(\Omega)}$, and take $\eta_{\varepsilon}(x, t)=\operatorname{sign} u_{\varepsilon}\left(\left|u_{\varepsilon}\right|-k\right)_{+} \chi_{(0, \tau)}(t)$ as a test function in problem $\left(P_{\varepsilon}\right)$, 
we obtain

$$
\begin{aligned}
\left\langle\frac{\partial u_{\varepsilon}}{\partial t},\right. & \left.\operatorname{sign} u_{\varepsilon}\left(\left|u_{\varepsilon}\right|-k\right)_{+} \chi_{(0, \tau)}(t)\right\rangle+\int_{0}^{\tau} \int_{\Omega} \bar{a}\left(x, t, u_{\varepsilon}, \nabla u_{\varepsilon}\right) \nabla\left(\operatorname{sign} u_{\varepsilon}\left(\left|u_{\varepsilon}\right|-k\right)_{+}\right) \mathrm{d} x \mathrm{~d} t \\
& +\int_{0}^{\tau} \int_{\Omega} \bar{\phi}\left(u_{\varepsilon}\right) \nabla\left(\operatorname{sign} u_{\varepsilon}\left(\left|u_{\varepsilon}\right|-k\right)_{+}\right) \mathrm{d} x \mathrm{~d} t \\
& +\int_{0}^{\tau} \int_{\Omega} g_{\varepsilon}\left(x, t, u_{\varepsilon}\right) \operatorname{sign} u_{\varepsilon}\left(\left|u_{\varepsilon}\right|-k\right)_{+} \mathrm{d} x \mathrm{~d} t \\
= & \int_{0}^{\tau} \int_{\Omega}\left(f_{\varepsilon}+\operatorname{div} F_{\varepsilon}\right) \operatorname{sign} u_{\varepsilon}\left(\left|u_{\varepsilon}\right|-k\right)_{+} \mathrm{d} x \mathrm{~d} t .
\end{aligned}
$$

Firstly, by Young's inequality and the Poincaré inequality, we get for any $\delta>0$

$$
\begin{aligned}
& \left|\int_{0}^{\tau} \int_{\Omega}\left(f_{\varepsilon}+\operatorname{div} F_{\varepsilon}\right) \operatorname{sign} u_{\varepsilon}\left(\left|u_{\varepsilon}\right|-k\right)_{+} \mathrm{d} x \mathrm{~d} t\right| \\
& \quad \leq C(\delta)\left\|f_{\varepsilon}+\operatorname{div} F_{\varepsilon}\right\|_{L^{\infty}(Q)} \psi_{\varepsilon}(k)+\delta \int_{0}^{T} \int_{A_{k \varepsilon}(t)}\left|\nabla\left(\left|u_{\varepsilon}\right|-k\right)_{+}\right|^{p^{-}} \mathrm{d} x \mathrm{~d} t,
\end{aligned}
$$

where $A_{k \varepsilon}(t)=\{x \in \Omega:|u(x, t)|>k\}$ and $\psi_{\varepsilon}(k)=\int_{0}^{T}$ meas $A_{k \varepsilon}(t) \mathrm{d} t$.

Secondly, it is easy to see that estimates (3.11)-(3.14) still hold with $u_{\varepsilon}$ instead of $u, A_{k \varepsilon}(t)$ instead of $A_{k}(t)$, and $\psi_{\varepsilon}(k)$ instead of $\psi(k)$. Hence, taking $\delta$ small enough in (3.59) and then applying all these results in (3.58), we get

$$
\sup _{0 \leq \tau \leq T} \int_{\Omega}\left[\left(\left|u_{\varepsilon}\right|-k\right)_{+}\right]^{2}(\tau) \mathrm{d} x+\int_{0}^{T} \int_{A_{k}(t)}\left|\nabla\left(\left|u_{\varepsilon}\right|-k\right)_{+}\right|^{p^{-}} \mathrm{d} x \mathrm{~d} t \leq C_{\varepsilon} \psi_{\varepsilon}(k),
$$

where $C_{\varepsilon}=C\left(\alpha, p^{-}, \Omega, N,\left\|f_{\varepsilon}+\operatorname{div} F_{\varepsilon}\right\|_{L^{\infty}(Q)}\right)$. Therefore, for $l \geq k$, by Proposition 3.1 of [18] we have

$$
\begin{aligned}
(l-k)\left(\psi_{\varepsilon}(l)\right)^{\frac{N}{p^{-}(N+2)}} & \leq\left(\int_{Q}\left|\left(\left|u_{\varepsilon}\right|-k\right)_{+}\right|^{\frac{p^{-}(N+2)}{N}} \mathrm{~d} x \mathrm{~d} t\right)^{\frac{N}{p^{-}(N+2)}} \\
& \leq C_{\varepsilon 1}\left(\psi_{\varepsilon}(k)\right)^{\frac{N+p^{-}}{p^{-(N+2)}}}
\end{aligned}
$$

where $C_{\varepsilon 1}$ is a positive constant. Taking $k=\left\|u_{0}\right\|_{L^{\infty}(\Omega)}$, we obtain

$$
\left(\psi_{\varepsilon}(l)\right)^{\frac{N}{p^{-(N+2)}}} \leq \frac{1}{l-\left\|u_{0}\right\|_{L^{\infty}(\Omega)}} C_{\varepsilon 1}|Q| .
$$

Then it follows that there exists a constant $\sigma_{\varepsilon}>1$ such that

$$
\psi_{\varepsilon}(l) \leq 2^{\frac{(N+2)\left(N+p^{-}\right)}{p^{-}}} \quad \text { for any } l \geq \sigma_{\varepsilon}+\left\|u_{0}\right\|_{L^{\infty}(\Omega)} .
$$

Let us consider the sequence $k_{n}=M_{\varepsilon}\left(2-2^{-n}\right)$, where $M_{\varepsilon}=\max \left\{\sigma_{\varepsilon}+\left\|u_{0}\right\|_{L^{\infty}(\Omega)}, C_{\varepsilon 1}\right\}$. Replacing $l, k$ by $k_{n+1}, k_{n}$ in (3.60), respectively, it then follows that

$$
\left(k_{n+1}-k_{n}\right)\left(\psi_{\varepsilon}\left(k_{n+1}\right)\right)^{\frac{N}{p^{-(N+2)}}} \leq C_{\varepsilon 1}\left(\psi_{\varepsilon}\left(k_{n}\right)\right)^{\frac{N+p^{-}}{p^{-(N+2)}}},
$$


which implies that

$$
\begin{aligned}
\psi_{\varepsilon}\left(k_{n+1}\right) & \leq\left(\frac{2 C_{\varepsilon 1}}{M_{\varepsilon}}\right)^{\frac{p^{-}(N+2)}{N}} 2^{\frac{n p^{-}(N+2)}{N}}\left(\psi_{\varepsilon}\left(k_{n}\right)\right)^{1+\frac{p^{-}}{N}} \\
& \leq 2^{\frac{p^{-}(N+2)}{N}} 2^{\frac{n p^{-}(N+2)}{N}}\left(\psi_{\varepsilon}\left(k_{n}\right)\right)^{1+\frac{p^{-}}{N}}
\end{aligned}
$$

Obviously, (3.61) holds for $l=k_{0}$. Hence, by Lemma 3.4 and (3.62), we have $u_{\varepsilon} \in L^{\infty}(Q)$ such that $\left\|u_{\varepsilon}\right\|_{L^{\infty}(Q)} \leq M_{\varepsilon}$.

Since $u_{\varepsilon} \in L^{\infty}(Q) \cap C\left([0, T] ; L^{2}(\Omega)\right)$ is a weak solution of $\left(P_{\varepsilon}\right)$, using the same argument of Lemma 3.3 we get

$$
\left\|u_{\varepsilon}\right\|_{L^{\infty}(Q)} \leq M,
$$

where $M$ is defined as before.

Step 3: In view of (3.63), we have

$$
\bar{a}\left(x, t, u_{\varepsilon}, \nabla u_{\varepsilon}\right)=a\left(x, t, u_{\varepsilon}, \nabla u_{\varepsilon}\right) \quad \text { and } \quad \bar{\phi}\left(u_{\varepsilon}\right)=\phi\left(u_{\varepsilon}\right) .
$$

Choosing $u_{\varepsilon}$ as a test function in $\left(P_{\varepsilon}\right)$, it follows from $\left(\mathrm{H}_{1}\right)-\left(\mathrm{H}_{6}\right)$ that

$$
\frac{1}{2}\left\|u_{\varepsilon}(t)\right\|_{L^{2}(\Omega)}^{2}+\alpha \int_{0}^{\tau} \int_{\Omega}\left|\nabla u_{\varepsilon}\right|^{p(x, t)} \mathrm{d} x \mathrm{~d} t \leq C, \quad \forall \tau \in(0, T],
$$

where $C$ is a positive constant independent of $\varepsilon$.

Hence, arguing as before, up to subsequences (still denoted by $\left\{u_{\varepsilon}\right\}$ ), we infer that

$$
\nabla u_{\varepsilon} \rightarrow \nabla u \quad \text { weakly in }\left(L^{p(x, t)}(Q)\right)^{N} \text { and weakly* in } L^{\infty}(Q)
$$

and

$$
u_{\varepsilon} \rightarrow u \quad \text { weakly in } L^{p^{-}}\left(0, T ; W_{0}^{1, p(x, t)}(\Omega)\right)
$$

Moreover, we have

$$
\frac{\partial u_{\varepsilon}}{\partial t} \text { is bounded in } W^{\star}(Q)+L^{1}(Q) \text { with respect to } \varepsilon \text {, }
$$

which implies that

$$
\frac{\partial u_{\varepsilon}}{\partial t} \text { is bounded in } L^{1}\left(0, T ;\left(W_{0}^{1, \lambda}(\Omega)\right)^{\star}\right) \text { with respect to } \varepsilon \text {, for } \lambda>N \text {. }
$$

Then the same argument of (3.36) shows that for subsequences of $\left\{u_{\varepsilon}\right\}$ (still denoted by $\left.\left\{u_{\varepsilon}\right\}\right)$,

$$
u_{\varepsilon} \rightarrow u \quad \text { a.e. in } Q \text {. }
$$


Proceeding as in the proof of (3.53), we get

$$
a\left(x, t, u_{\varepsilon}, \nabla u_{\varepsilon}\right) \rightarrow a(x, t, u, \nabla u) \quad \text { strongly in }\left(L^{p^{\prime}(x, t)}(Q)\right)^{N} .
$$

Obviously, we also obtain $u \in W(Q) \cap L^{\infty}(Q)$ and $\frac{\partial u}{\partial t} \in W^{\star}(Q)+L^{1}(Q)$, thus $u \in$ $C\left([0, T] ; L^{2}(\Omega)\right)$. Furthermore, we get $\left.u\right|_{t=0}=u_{0}$. Thus, let $\varepsilon \rightarrow 0$ in $\left(P_{\varepsilon}\right)$, with the help of (3.63)- (3.68) and assumptions $\left(\mathrm{H}_{1}\right)-\left(\mathrm{H}_{6}\right)$, we deduce that $u$ is a solution to problem $(P)$.

\section{Uniqueness of weak solutions to problem $(P)$}

In order to get the uniqueness result, we need the following assumptions:

$\left(\mathrm{H}_{7}\right)$ The function $\phi$ is locally Lipschitz continuous.

$\left(\mathrm{H}_{8}\right)$ For every $k>0$, there exist $\bar{c}_{k} \in L^{p^{\prime}(x, t)}(Q)$ and a constant $\beta_{k}>0$ such that

$$
\begin{aligned}
& \left|a\left(x, t, s_{1}, \xi\right)-a\left(x, t, s_{2}, \xi\right)\right| \\
& \quad \leq\left|s_{1}-s_{2}\right|\left[\beta_{k}|\xi|^{p(x, t)-1}+\bar{c}_{k}(x, t)\right], \quad \text { a.e. }(x, t) \in Q,
\end{aligned}
$$

for every $\forall \xi \in \mathbb{R}^{N}$ and every $\left|s_{1}\right| \leq k$ and $\left|s_{2}\right| \leq k$.

$\left(\mathrm{H}_{9}\right) g: \Omega \times[0, T] \times \mathbb{R}$ is monotone with respect to the third variable.

Theorem 4.1 Assume $p \in C_{+}(\bar{\Omega})$ and the conditions $\left(\mathrm{H}_{1}\right)-\left(\mathrm{H}_{9}\right)$ hold, then problem $(P)$ admits a unique weak solution $u(x, t) \in W \cap C\left([0, T] ; L^{2}(\Omega)\right) \cap L^{\infty}(Q)$.

Proof of Theorem 4.1 The existence result is proved by Theorem 3.1. In the following, we prove the uniqueness result. Assume that $u, v \in W \cap C\left([0, T] ; L^{2}(\Omega)\right) \cap L^{\infty}(Q)$ are two weak solutions of $(P)$, then taking $\eta=\frac{1}{\varepsilon} T_{\varepsilon}(u-v) \chi_{(0, \tau)(t)}$, the following equality holds:

$$
\begin{gathered}
\int_{0}^{T}\left\langle\frac{\partial u}{\partial t}, \eta\right\rangle \mathrm{d} \tau+\int_{0}^{T} \int_{0}^{\tau} \int_{\Omega}[a(x, t, u, \nabla u)+\phi(u)] \nabla \eta \mathrm{d} x \mathrm{~d} t \mathrm{~d} \tau \\
+\int_{0}^{T} \int_{0}^{\tau} \int_{\Omega} g(x, t, u) \eta \mathrm{d} x \mathrm{~d} t \mathrm{~d} \tau \\
=\int_{0}^{T} \int_{0}^{\tau} \int_{\Omega} f \eta \mathrm{d} x \mathrm{~d} t \mathrm{~d} \tau+\int_{0}^{T} \int_{0}^{\tau} \int_{\Omega} F \nabla \eta \mathrm{d} x \mathrm{~d} t \mathrm{~d} \tau, \\
\int_{0}^{T}\left\langle\frac{\partial v}{\partial t}, \eta\right\rangle \mathrm{d} \tau+\int_{0}^{T} \int_{0}^{\tau} \int_{\Omega}[a(x, t, v, \nabla v)+\phi(v)] \nabla \eta \mathrm{d} x \mathrm{~d} t \mathrm{~d} \tau \\
+\int_{0}^{T} \int_{0}^{\tau} \int_{\Omega} g(x, t, v) \eta \mathrm{d} x \mathrm{~d} t \mathrm{~d} \tau \\
=\int_{0}^{T} \int_{0}^{\tau} \int_{\Omega} f \eta \mathrm{d} x \mathrm{~d} t \mathrm{~d} \tau+\int_{0}^{T} \int_{0}^{\tau} \int_{\Omega} F \nabla \eta \mathrm{d} x \mathrm{~d} t \mathrm{~d} \tau .
\end{gathered}
$$

Subtracting equality (4.3) from (4.2) and using Lemma 3.2, we obtain

$$
\begin{aligned}
& \frac{1}{\varepsilon} \int_{0}^{T} \int_{\Omega} \tilde{T}_{\varepsilon}(u(x, \tau)-v(x, \tau)) \mathrm{d} x \mathrm{~d} \tau \\
& \quad+\frac{1}{\varepsilon} \int_{0}^{T} \int_{0}^{\tau} \int_{\Omega}[a(x, t, u, \nabla u)-a(x, t, v, \nabla v)] \nabla T_{\varepsilon}(u-v) \mathrm{d} x \mathrm{~d} t \mathrm{~d} \tau
\end{aligned}
$$




$$
\begin{aligned}
& +\frac{1}{\varepsilon} \int_{0}^{T} \int_{0}^{\tau} \int_{\Omega}[\phi(u)-\phi(v)] \nabla T_{\varepsilon}(u-v) \mathrm{d} x \mathrm{~d} t \mathrm{~d} \tau \\
& +\frac{1}{\varepsilon} \int_{0}^{T} \int_{0}^{\tau} \int_{\Omega}[g(x, t, u)-g(x, t, v)] T_{\varepsilon}(u-v) \mathrm{d} x \mathrm{~d} t \mathrm{~d} \tau=0,
\end{aligned}
$$

where $\tilde{T}_{\varepsilon}(r)=\int_{0}^{r} T_{\varepsilon}(s) \mathrm{d} s$.

Denote the four terms on the left hand side by $L_{1}(\varepsilon), L_{2}(\varepsilon), L_{3}(\varepsilon)$, and $L_{4}(\varepsilon)$, respectively. Concerning the first term $L_{1}(\varepsilon)$, we have

$$
\lim _{\varepsilon \rightarrow 0} L_{1}(\varepsilon)=\int_{0}^{T} \int_{\Omega}|u(x, \tau)-v(x, \tau)| \mathrm{d} x \mathrm{~d} \tau
$$

For the second term $L_{2}(\varepsilon)$, in view of $\left(\mathrm{H}_{3}\right)$ we get

$$
\begin{aligned}
L_{2}(\varepsilon)= & \frac{1}{\varepsilon} \int_{0}^{T} \int_{0}^{\tau} \int_{\Omega}[a(x, t, u, \nabla u)-a(x, t, v, \nabla u)] \nabla T_{\varepsilon}(u-v) \mathrm{d} x \mathrm{~d} t \mathrm{~d} \tau \\
& +\frac{1}{\varepsilon} \int_{0}^{T} \int_{0}^{\tau} \int_{\Omega}[a(x, t, v, \nabla u)-a(x, t, v, \nabla v)] \nabla T_{\varepsilon}(u-v) \mathrm{d} x \mathrm{~d} t \mathrm{~d} \tau \\
\geq & \frac{1}{\varepsilon} \int_{0}^{T} \int_{0}^{\tau} \int_{\Omega}[a(x, t, u, \nabla u)-a(x, t, v, \nabla u)] \nabla T_{\varepsilon}(u-v) \mathrm{d} x \mathrm{~d} t \mathrm{~d} \tau
\end{aligned}
$$

By $\left(\mathrm{H}_{8}\right)$, we obtain

$$
\begin{aligned}
& \left|\frac{1}{\varepsilon} \int_{0}^{T} \int_{0}^{\tau} \int_{\Omega}[a(x, t, u, \nabla u)-a(x, t, v, \nabla u)] \nabla T_{\varepsilon}(u-v) \mathrm{d} x \mathrm{~d} t \mathrm{~d} \tau\right| \\
& \quad \leq T \int_{\{|u-v| \leq \varepsilon\} \cap\{u \neq v\}}\left[\beta_{M}|\nabla u|^{p(x, t)-1}+\bar{c}_{M}(x, t)\right][|\nabla u|+|\nabla v|] \mathrm{d} x \mathrm{~d} t
\end{aligned}
$$

where $M$ is defined as Lemma 3.3.

Note that

$$
\chi_{\{|u-v| \leq \varepsilon\} \cap\{u \neq v\}} \rightarrow 0 \quad \text { a.e. in } Q
$$

using Lebesgue's dominated convergence theorem, we find that

$$
\varlimsup_{\varepsilon \rightarrow 0}\left|\frac{1}{\varepsilon} \int_{0}^{T} \int_{0}^{\tau} \int_{\Omega}[a(x, t, u, \nabla u)-a(x, t, v, \nabla u)] \nabla T_{\varepsilon}(u-v) \mathrm{d} x \mathrm{~d} t \mathrm{~d} \tau\right|=0 .
$$

Combining (4.6) with (4.8), we obtain

$$
\varlimsup_{\varepsilon \rightarrow 0} L_{2}(\varepsilon) \geq 0
$$

Similarly to the proof of (4.8), using $\left(\mathrm{H}_{7}\right)$ we have

$$
\varlimsup_{\varepsilon \rightarrow 0} L_{3}(\varepsilon)=0
$$

Condition $\left(\mathrm{H}_{9}\right)$ implies that

$$
L_{4}(\varepsilon) \geq 0 .
$$


Let $\varepsilon \rightarrow 0$ in (4.4), it follows from (4.5) and (4.9)-(4.11) that

$$
\int_{0}^{T} \int_{\Omega}|u(x, \tau)-v(x, \tau)| \mathrm{d} x \mathrm{~d} \tau=0
$$

Hence, we have $u=v$ a.e. in $Q$.

\section{Appendix}

Proof of Lemma 3.2 The proof is similar to the proof of Lemma 7.1 in [29], but we use another approximation here. For the sake of clarity and readability, we give the details below.

First of all, by Remark 3.2, we note $u \in C\left([0, T] ; L^{1}(\Omega)\right)$. We take the Steklov average of the function $u$ by

$$
u_{h}=\frac{1}{h} \int_{t}^{t+h} u(x, \tau) \mathrm{d} \tau
$$

Appropriately extending the functions $u$ outside $(0, T)$, we still get $u_{h} \in W(Q)$ with $\frac{\partial u}{\partial t} \in$ $W(Q)$ and convergence, as $h \rightarrow 0$ strongly to $u$ in $W(Q)$ and a.e. in $Q$. By the properties of $\varphi$ and Remark 3.2, we also get $\varphi\left(u_{h}\right) \in W(Q) \cap C\left([0, T] ; L^{1}(\Omega)\right)$ and $\varphi\left(u_{h}\right) \rightarrow \varphi(u)$ strongly in $W(Q)$. Hence we have

$$
\begin{aligned}
& \int_{0}^{T}\left\langle\frac{\partial u_{h}}{\partial t}, \varphi\left(u_{h}\right) \chi_{(0, \tau)(t)} \psi\right\rangle \mathrm{d} t \\
& \quad=\int_{0}^{\tau} \int_{\Omega} \frac{\partial u_{h}}{\partial t} \varphi\left(u_{h}\right) \psi \mathrm{d} x \mathrm{~d} t=\int_{0}^{\tau} \int_{\Omega} \frac{\partial \tilde{\varphi}\left(u_{h}\right)}{\partial t} \psi \mathrm{d} x \mathrm{~d} t \\
& \quad=\left.\int_{\Omega}\left(\tilde{\varphi}\left(u_{h}\right) \psi\right)\right|_{t=\tau} \mathrm{d} x-\left.\int_{\Omega}\left(\tilde{\varphi}\left(u_{h}\right) \psi\right)\right|_{t=0} \mathrm{~d} x-\int_{0}^{\tau} \int_{\Omega} \frac{\partial \psi}{\partial t} \tilde{\varphi}\left(u_{h}\right) \mathrm{d} x \mathrm{~d} \tau
\end{aligned}
$$

which yields

$$
\begin{aligned}
& \int_{0}^{T}\left\langle\frac{\partial u}{\partial t}, \varphi(u(t)) \chi_{(0, \tau)(t)} \psi\right\rangle \mathrm{d} t \\
& \quad=\lim _{h \rightarrow 0} \int_{0}^{T}\left\langle\frac{\partial u_{h}}{\partial t}, \varphi\left(u_{h}\right) \chi_{(0, \tau)(t)} \psi\right\rangle \mathrm{d} t \\
& \quad=\left.\int_{\Omega}(\tilde{\varphi}(u) \psi)\right|_{t=\tau} \mathrm{d} x-\left.\int_{\Omega}(\tilde{\varphi}(u) \psi)\right|_{t=0} \mathrm{~d} x-\int_{0}^{\tau} \int_{\Omega} \frac{\partial \psi}{\partial t} \tilde{\varphi}(u) \mathrm{d} x \mathrm{~d} \tau .
\end{aligned}
$$

Thus the assertion of the lemma follows.

\section{Competing interests}

The authors declare that they have no competing interests.

Authors' contributions

All authors contributed equally to the manuscript and read and approved the final manuscript.

\section{Acknowledgements}

The authors wish to thank the referees for careful reading of the early version of this manuscript and providing valuable corrections, suggestions and comments. This work is supported by the NSFC of China (no. 11461048), the Natural Science Foundation of JiangXi Province of China (no. 20132BAB211006), the foundation of Jiangxi Educational Committee (no. GJJ14546), and NSFC of China (no. 11126338). 
Received: 19 November 2014 Accepted: 14 April 2015 Published online: 25 April 2015

\section{References}

1. Rüžička, M: Electrorheological Fluids: Modelling and Mathematical Theory. Lecture Notes in Mathematics, vol. 1748. Springer, Berlin (2000)

2. Antontsev, SN, de Oliveira, HB: Asymptotic behavior of trembling fluids. Nonlinear Anal., Real World Appl. 19, 54-66 (2014)

3. Acerbi, E, Mingione, G: Regularity results for stationary electro-rheological fluids. Arch. Ration. Mech. Anal. 164, 213-259 (2002)

4. Chen, Y, Levine, S, Rao, M: Variable exponent, linear growth functionals in image restoration. SIAM J. Appl. Math. 66, 1383-1406 (2006)

5. Zhikov, V: Averaging of functionals of the calculus of variations and elasticity theory. IzV. Akad. Nauk SSSR, Ser. Mat. 50(4), 675-710 (1986)

6. Pucci, P, Zhang, Q: Existence of entire solutions for a class of variable exponent elliptic equations. J. Differ. Equ. 257(5), 1529-1566 (2014)

7. Bendahmane, M, Wittbold, P: Renormalized solutions for nonlinear elliptic equations with variable exponents and $L^{1}$ data. Nonlinear Anal. 70, 567-583 (2009)

8. Sanchón, M, Urbano, JM: Entropy solutions for the $p(x)$-Laplace equation. Trans. Am. Math. Soc. 361, 6387-6405 (2009)

9. LV, B, Li, F, Zou, W: Existence of weak solutions for some nonlinear elliptic equations with variable exponents. Complex Var. Elliptic Equ. 58(10), 1431-1447 (2013)

10. Zhang, X, Fu, Y: Solutions for nonlinear elliptic equations with variable growth and degenerate coercivity. Ann. Mat. Pura Appl. 193(1), 133-161 (2014)

11. Zou, W, Wang, W, Bi, Y: On a class of nonlinear obstacle problems with nonstandard growth. Math. Method. Appl. Sci. (in press). doi:10.1002/mma.3250

12. Zhang, C, Zhou, S: Renormalized and entropy solutions for nonlinear parabolic equations with variable exponents and $L^{1}$ data. J. Differ. Equ. 248, 1376-1400 (2010)

13. Bendahmane, M, Wittbold, P, Zimmermann, A: Renormalized solutions for a nonlinear parabolic equation with variable exponents and $L^{1}$-data. J. Differ. Equ. 249, 1483-1515 (2010)

14. Lian, S, Gao, W, Yuan, H, Cao, C: Existence of solutions to an initial Dirichlet problem of evolutional $p(x)$-Laplace equations. Ann. Inst. Henri Poincaré, Anal. Non Linéaire 29(3), 377-399 (2012)

15. Acerbi, E, Mingione, G, Seregin, GA: Regularity results for parabolic systems related to a class of non-Newtonian fluids. Ann. Inst. Henri Poincaré, Anal. Non Linéaire 21, 25-60 (2004)

16. Antontsev, S, Shmarev, S: Anisotropic parabolic equations with variable nonlinearity. Publ. Mat. 53(2), 355-399 (2009)

17. Alkhutov, YA, Zhikov, W: Existence theorems for solutions of parabolic equations with a variable order of nonlinearity. Proc. Steklov Inst. Math. 270(1), 15-26 (2010)

18. Dibenedetto, E: Degenerate Parabolic Equations. Springer, New York (1993)

19. Zou, W, Wang, W: Existence of solutions for a class of porous medium type equations with $L^{1}$ data. Nonlinear Anal. 98 , 165-189 (2014)

20. Fan, $X$, Zhao, D: On the spaces $L^{p(x)}(\Omega)$ and $W^{m, p(x)}(\Omega)$. J. Math. Anal. Appl. 263, 424-446 (2001)

21. Diening, L, Harjulehto, $P$, Hästö, P, Ruzicka, M: Lebesgue and Sobolev Spaces with Variable Exponents. Lecture Notes in Mathematics, vol. 2017 (2011)

22. Harjulehto, $\mathrm{P}$, Hästö, P, Koskenoja, M, Varonen, S: The Dirichlet energy integral and variable exponent Sobolev spaces with zero boundary values. Potential Anal. 25(3), 205-222 (2006)

23. Wittbold, P, Zimmermann, A: Existence and uniqueness of renormalized solutions to nonlinear elliptic equations with variable exponents and $L^{1}$-data. Nonlinear Anal. 72, 2990-3008 (2010)

24. Adams, R: Sobolev Spaces. Pure and Applied Mathematics, vol. 65. Academic Press, New York (1975)

25. Porretta, A: Existence results for nonlinear parabolic equations via strong convergence of truncations. Ann. Mat. Pura Appl. 177(4), 143-172 (1999)

26. Simon, J: Compact sets in $L^{P}(0, T ; B)$. Ann. Mat. Pura Appl. 146, 65-96 (1987)

27. Landes, R: On the existence of weak solution for quasilinear parabolic initial boundary-value problems. Proc. R. Soc. Edinb. A 89, 217-237 (1981)

28. Boccardo, L, Murat, F, Puel, JP: Existence of bounded solutions for nonlinear elliptic unilateral problems. Ann. Mat. Pura Appl. 152(4), 183-196 (1988)

29. Droniou, J, Prignet, A: Equivalence between entropy and renormalized solutions for parabolic equations with smooth measure data. NoDEA Nonlinear Differ. Equ. Appl. 14, 181-205 (2007) 